\title{
A fast generalizable solution method for glucose control algorithms
}

\author{
C.E. Hann ${ }^{1}$, P. Docherty ${ }^{1}$, J.G. Chase ${ }^{1}$ and G. M. Shaw ${ }^{2}$
}

Department of Mechanical Engineering

University of Canterbury

Private Bag 4800

Christchurch

New Zealand

Email: Chris.Hann@canterbury.ac.nz

\footnotetext{
${ }^{1}$ Department of Mechanical Engineering, University of Canterbury, Christchurch, New Zealand

${ }^{2}$ Department of Intensive Care, Christchurch Hospital, Christchurch, New Zealand
}

Note to readers. For the definitive version of this document, refer to:

Hann, C.E., Docherty, P., Chase, J.G. and Shaw, G.M. (2010) A fast generalizable solution method for glucose control algorithms. Mathematical Biosciences, 227(1), 44-55.

http://dx.doi.org/10.1016/i.mbs.2010.06.001 


\begin{abstract}
In critical care tight control of blood glucose levels has been shown to lead to better clinical outcomes. The need to develop new protocols for tight glucose control, as well as the opportunity to optimize a variety of other drug therapies, has led to a resurgence in model-based medical decision support in this area. One still valid hindrance to developing new model-based protocols using so-called virtual patients, retrospective clinical data, and Monte Carlo methods is the large amount of computational time and resources needed.
\end{abstract}

This paper develops fast analytical-based methods for an insulin-glucose system model that are generalizable to other similar systems. Exploiting the structure and partial solutions in a subset of the model is the key in finding accurate fast solutions to the full model. This approach successfully reduced computing time by factors of 5600-144000 depending on the numerical error management method, for large (50-164 patient) virtual trials and Monte Carlo analysis. It thus allows new model-based or model-derived protocols to be rapidly developed via extensive simulation. The new method is rigorously compared to existing standard numerical solutions and is found to be highly accurate to within $0.2 \%$.

Keywords: Model-based therapeutics; fast simulation; blood glucose control; Monte Carlo analysis; analytical solutions; computation

\title{
$1 \quad$ Introduction
}

Fast and efficient computational methods are important for real-time therapy guidance and are a growing trend in Bio-Engineering [1-11]. More recently, significant clinical outcomes have been achieved by table-based glucose control protocols [12-14] that mimic and are developed from computer-based protocols [15-18], but are much simpler to implement and more likely to be accepted by clinical staff [19]. Such model-derived or model-validated approaches are also increasing in prevalence [20, 21]. 
Tight control of blood glucose levels gained significant importance in critical care after Van den Berghe et al. [22] showed reductions in mortality rates of up to $45 \%$ are possible. The common way of assessing risk of death in the ICU is by an APACHE score, which measures the severity of illness. Thus, higher scores mean a higher risk of death. The trials in [12-14] represent the first model-based or model-derived protocol to report a significant clinical outcome with a 32\% mortality reduction [18], despite significantly high APACHE scores. This trial also had some of the tightest glucose control reported as compared to other studies e.g. [2, 18, 23-29], indicating the potential of computational models to impact this field.

A major component in the development of these model-based protocols $[13,14,18]$ is the concept of "virtual patient trials" where each new potential protocol is tested in simulation off-line, with up to hundreds of thousands of patient hours $[12,21]$. This approach can include Monte Carlo analysis methods, which can add the effects of parameter uncertainty and different sensor errors into the analysis [16, 30]. The major advantage of a "virtual patient" approach is that algorithms and methods can be tested and optimized safely before clinical implementation. Furthermore, the approach gives insight into potential long term clinical performance and provides a repeatable cohort for easy comparison of different protocols $[12,13]$. The considerable patient variation and sensor noise combined with different variations in the glucose-insulin physiological modelling can lead to large numbers of simulations required. Thus, a fundamental requirement in this "model-based therapeutics" approach is very fast and accurate simulation methods that can be performed using simple programme languages, such as Matlab, to minimize development time.

A virtual patient database represents a summary of the metabolic changes and interventions for patients in a given cohort over the entire length of stay in the intensive care unit (ICU). The primary parameter used to represent patients' metabolic status [12, 13] was insulin sensitivity $\left(S_{I}\right)$, which was allowed to vary with time but was held constant over each one hour period. These $S_{I}$ profiles were established from 
retrospective clinical data using an integral-based parameter identification method [20]. Data from patients in other trials [15-18] was also added to this database.

The process of creating a virtual patient cohort and then testing or creating new protocols using the physiological model is referred to here as "model-based therapeutics" (MBT). This MBT approach led to the development of the Specialized Relative Insulin + Nutrition Tables (SPRINT) protocol that has been implemented in the Christchurch Hospital ICU [12-14, 18, 31]. Successful initial results have also been obtained in several other areas, including model based cardiac diagnosis [32-36], sedative drug delivery [37, 38] and insulin sensitivity measurement [30, 39].

In all these cases, extensive simulation (Monte Carlo based) is performed in virtual trials to ensure robustness of the protocols developed. Some analyses run as many as 100,000200,000 patient hours (80-160 patient years) in total [13, 14]. Thus, a fundamental element in the MBT approach must be highly accurate, easily programmable, very fast forward solutions to non-linear physiological models to reduce computational burden and run-time.

To get an idea of the computation time required, the original MATLAB simulation code used to develop the SPRINT system [13, 14], which originally used 19 patients, takes 0.9 seconds to simulate one patient hour on a PC with a $2.4 \mathrm{GHz}$ processor and 2GB RAM. This simulation uses a maximum step size of 0.019 based on an error analysis to ensure the $90^{\text {th }}$ percentile error is less than $0.1 \%$. Note that using an error analysis based on reducing the relative error tolerance instead of the maximum step size, would yield faster results of $0.035 \mathrm{~s}$ for one patient hour. However, for highly stiff systems (e.g. [36]), the maximum step size method was found to be a more reliable and conservative option, so was used in the development of SPRINT. In this case, the Monte Carlo (100x) testing of a new protocol on 384 patients (42,000 hours) would require 4,200,000 patient hours $\times$ $0.9 \mathrm{~s}=43.8$ days of simulation. As more patients are added, greater numbers of protocols are tested, and potentially more dynamics are added to the glucose-insulin model (e.g. a more detailed carbohydrate input model), then even more significant computing time and 
resources would be required. Thus, the computational time would significantly limit the number of patients and protocols that could be tested, potentially hindering research and clinical outcomes.

The glucose control protocols discussed [2, 12-17, 40, 41] require a simulated measurement only 1-2 hourly. In contrast, to obtain sufficient accuracy, numerical ODE solvers (e.g. [16]) compute the glucose value at every time step (minutes or seconds) throughout an hour. In other words, a large percentage of computation is spent calculating glucose values that are not used in a virtual trial or clinical control. However, the reasonably typical non-linear glucose-insulin model [17] has no direct analytical solution. Furthermore, other insulin-glucose models reported in the literature, ranging from relatively simple e.g. [42], to far more complicated [43], also require computationally intense numerical solutions. Hence, the computational cost of extensive virtual trials simulations yields values that are necessary only for accurate solution by the solver, but are not used directly in protocol development.

The approach in this research, is to form partial analytical solutions to a subset of the model in terms of simpler approximating functions that are valid within known physiological bounds. These simpler functions then enable an analytical solution to the whole model to be constructed. More specifically, the approach is to reformulate and tailor solutions to the specific application required. With careful construction and the use of known mathematical solutions to differential equations, it is shown that an approximate, but highly accurate, analytical solution can be found to the full non-linear glucose-insulin system model. This solution dramatically increases the forward simulation speed of the model, by avoiding the need to calculate values that are not used in the virtual trial simulations. It thus enables far more rapid development of new protocols using the virtual patient based MBT approach. 


\section{$2 \quad$ Methodology}

\subsection{Model of glucose and insulin kinetics}

The model of glucose and insulin kinetics [17] used to run virtual trials in this research originates from Bergman's minimal model [42]. Additional non-linear terms, a more physiologically representative formulation, and time varying insulin sensitivity, $S_{I}=S_{I}(t)$, creates the system model defined [2, 12-17, 40]:

$$
\begin{gathered}
\dot{G}=-p_{G} G-S_{I}\left(G+G_{e q}\right) \frac{Q}{1+\alpha_{G} Q}+P(t) \\
\dot{Q}=-k Q+k \mathrm{I} \\
\dot{I}=-\frac{n \mathrm{I}}{1+\alpha_{\mathrm{I}} \mathrm{I}}+\frac{u_{e x}}{V} \\
P\left(t_{i}<t<t_{i+1}\right)=\bar{P}_{i+1}+\left(P\left(t_{i}\right)-\bar{P}_{i+1}\right) e^{-k_{p}\left(t-t_{i}\right)} \\
k_{p}=\left\{\begin{array}{l}
k_{p d} \\
k_{p r}
\end{array}\right\} \text { if } \bar{P}_{i+1}<P\left(t_{i}\right) \\
\bar{P}_{i+1}>P\left(t_{i}\right)
\end{gathered}
$$

where $G_{e q}$ is the equilibrium plasma glucose concentration and $G$ is the plasma glucose concentration above $G_{e q}$. The exogenous feed input rate is denoted $P(t) ; I(t)$ is the plasma insulin resulting from exogenous insulin input $u_{e x}(t) ; Q(t)$ is the concentration of insulin bounded to interstitial sites; $k$ accounts for the effective life of insulin in the system. Patient endogenous glucose clearance and insulin sensitivity are $p_{G}$ and $S_{I}$ respectively; $V$ is the insulin distribution volume and $n$ is the constant first order decay rate for insulin from plasma. Michaelis-Menten functions are used to model saturation, with $\alpha_{I}$ used for the saturation of insulin disappearance, and $\alpha_{G}$ for the saturation of insulin-dependent glucose clearance; $k_{p r}$ and $k_{p d}$ are the decay constants for increasing and decreasing feed rates where $\bar{P}_{i}$ and $\bar{P}_{i+1}$ are the steps in enteral glucose feed rates. Thus, Equation (4) is essentially the analytical solution to the 2 compartment system in 
$[16,17]$. Note that $P(t)$ is defined as $\mathrm{mmol} / \mathrm{L} / \mathrm{min}$, accounting for the glucose distribution volume.

The parameters $\quad\left(V=12 \mathrm{~L}, \quad n=0.16 \mathrm{~min}^{-1}, \quad k=0.01 \mathrm{~min}^{-1}, \quad \alpha_{G}=0.04 \mathrm{~L} / \mathrm{mU}\right.$, $\alpha_{I}=0.0017 \mathrm{~L} / \mathrm{mU}, p_{G}=0.01 \mathrm{~min}^{-1}$ ) are average values found from an extensive literature search and $G_{e q}$ is estimated from the moving mean glucose concentration during a patient's stay $[17,20]$. The parameter $S_{I}$ is patient specific. Note that for simplicity, the model is reformulated by replacing $G$ by $G_{t o t a l}-G_{e q}$ in Equation (1):

$$
\dot{G}_{\text {total }}=-p_{G}\left(G_{\text {total }}-G_{e q}\right)-S_{I} G_{\text {total }} \frac{Q}{1+\alpha_{G} Q}+P(t)
$$

\subsection{Virtual patient cohort}

For this research, 17 uncontrolled patients from the study in [20] and 51 controlled patients in the original pilot study of the SPRINT system $[13,14]$ were used to form the virtual patient cohort. Each patient's physiology was summarized by their insulin sensitivity profile $S_{I}(t)$, which was obtained using the integral based parameter identification method of [20] and the retrospective data.

Given new nutritional and insulin inputs, the virtual patient's $S_{I}(t)$ profile can be used in Equations (2)-(5) to generate a (new) set of glucose levels for these different interventions, which is referred to as a "virtual trial” [12, 44]. Different protocols can then be applied on a specific patient by changing the nutritional and insulin inputs for the identified $S_{I}(t)$ profiles. Thus, potential improvements to a current retrospective protocol can be tested without requiring a full clinical trial. Therefore, for any new clinical trial, a protocol could be shown to be 'safe' and robust, as well as being optimized for the best possible clinical outcomes. 


\subsection{Analytical solution to insulin $I(t)$ without saturation}

In the ICU, for a given hour, the exogenous insulin input $u_{e x}(t)$ in Equation (3) is a combination of a bolus and infusion and is defined:

$$
\begin{aligned}
u_{e x}(t) & =u_{b}+u_{i}, \quad 0 \leq t \leq 1 \\
& =u_{i}, \quad 1<t \leq 60
\end{aligned}
$$

If the saturation parameter $\alpha_{I}$ is set to 0, Equation (3) has an analytical solution:

$$
\begin{aligned}
& \begin{array}{l}
I_{\alpha_{I}=0}(t)=I_{b}(t), \quad 0 \leq t \leq 1 \\
\quad=I_{i}(t), \quad 1 \leq t \leq 60
\end{array} \\
& I_{i}(t)=\frac{U_{i}}{n}+e^{-n(t-1)}\left(I_{b}(1)-\frac{U_{i}}{n}\right), \quad 1<t \leq 60 \\
& I_{b}(t)=\frac{1}{n}\left(u_{b}+u_{i}-e^{-n t}\left(u_{b}+u_{i}-n I_{0}\right)\right)
\end{aligned}
$$

The solution in Equations (7)-(9) will serve as an initial approximation to the solution of Equation (3) with $\alpha_{I} \neq 0$.

\subsection{Iterative analytical solution $I(t)$ with saturation}

For a given approximation, $I_{a p p r o x}(t)$ to $I(t)$, Equation (3) can be approximated by: 


$$
\begin{gathered}
\dot{I}=\alpha(t) I+u_{\text {ext }}(t) \\
\alpha(t)=\frac{n}{1+\alpha_{I} I_{\text {approx }}(t)}
\end{gathered}
$$

where $\alpha(t)$ is a known function of time. However, Equation (10) does not have a closed form analytical solution for any form of $I_{\text {approx }}(t)$. Thus, the approach is to approximate $\alpha(t)$ in Equation (11) by an integrable function, then Equation (10) can be solved by the integrating factor.

\subsubsection{Insulin solution in the first minute}

In the first minute, $I_{\text {approx }}(t)$ in Equation (11) is replaced by $I_{b}(t)$ of Equation (9), which yields:

$$
\alpha_{b}(t)=\frac{n}{1+\alpha_{I} I_{b}(t)}
$$

The integrating factor of Equation (10) with $\alpha(t)=\alpha_{b}(t)$ from Equation (12) is defined:

$$
\begin{gathered}
\mu_{b}(t)=e^{\text {int } \alpha_{b}(t)} \\
\operatorname{int}_{\alpha_{b}}(t)=\int_{o}^{t} \alpha_{b}(\tau) d \tau
\end{gathered}
$$

The analytical solution to Equation (10) in the first minute is thus:

$$
I_{b, a p p r o x}(t)=I_{0} e^{-i n \alpha_{\alpha_{b}}(t)}+e^{-i n \alpha_{b}(t)}\left(u_{i}+u_{b}\right) \int_{0}^{t} e^{\text {int } \alpha_{b}(\tau)} d \tau
$$


There is no closed form solution to the integral in Equation (14), therefore a piecewise linear approximation to int ${ }_{\alpha_{b}}(t)$ in Equation (14) over $t=0,0.5,1$ is defined:

$$
\begin{gathered}
\overline{\operatorname{int}}_{\alpha_{b}}(t)=b_{1} t, \quad 0 \leq t \leq \frac{1}{2} \\
=a_{2}+b_{2} t, \quad \frac{1}{2}<t \leq 1 \\
b_{1}=2 \text { int }_{\alpha_{b}}\left(\frac{1}{2}\right), a_{2}=2 \text { int }_{\alpha_{b}}\left(\frac{1}{2}\right)-\operatorname{int}_{\alpha_{b}}(1), b_{1}=2\left(\operatorname{int}_{\alpha_{b}}(1)-\operatorname{int}_{\alpha_{b}}\left(\frac{1}{2}\right)\right)
\end{gathered}
$$

where from the trapezium rule:

$$
\begin{aligned}
\operatorname{int}_{\alpha_{b}}\left(\frac{1}{2}\right) & =\frac{1}{4}\left(\alpha_{b}(0)+\alpha_{b}\left(\frac{1}{2}\right)\right) \\
\text { int }_{\alpha_{b}}(1) & =\operatorname{int}_{\alpha_{b}}\left(\frac{1}{2}\right)+\frac{1}{4}\left(\alpha_{b}\left(\frac{1}{2}\right)+\alpha_{b}(1)\right)
\end{aligned}
$$

Using Equation (16) to approximate int $\alpha_{b}(t)$, closed form expressions for the solution of Equation (15) at $t=\frac{1}{2}$ and $t=1$ are defined:

$$
\begin{aligned}
I_{b, \text { approx }}\left(\frac{1}{2}\right) & =I_{0} e^{-\frac{1}{2} b_{1}}+\frac{\left(u_{b}+u_{i}\right)}{b_{1}}\left(1-e^{-\frac{1}{2} b_{1}}\right) \\
I_{b, \text { approx }}(1) & =I_{b, \text { approx }}\left(\frac{1}{2}\right) e^{-a_{2}+\frac{1}{2} b_{1}-b_{2}}+\frac{\left(u_{b}+u_{i}\right)}{b_{2}}\left(1-e^{-\frac{1}{2} b_{2}}\right)
\end{aligned}
$$

where $a_{2}, b_{1}$ and $b_{2}$ are defined in Equation (17).

\subsubsection{Insulin solution after first minute}

After the first minute, $I_{a p p r o x}(t)$ in Equation (11) is replaced by $I_{\alpha_{I}=0}(t)$ in Equation (7), which yields: 


$$
\alpha_{i}(t)=\frac{n}{1+\alpha_{I} I_{\alpha_{I}=0}(t)}, 1 \leq t \leq 60
$$

To enable Equation (22) to be integrated analytically $\alpha_{i}(t)$ is approximated by the function:

$$
\begin{aligned}
& \begin{aligned}
\bar{\alpha}_{i}(t) & =a_{0}+\left(a_{1}+a_{2} t+a_{3} t^{2}\right) e^{-n t} \\
& =\alpha_{i}(1)+A_{1}\left(e^{-n(t-1)}-1\right)+A_{2}(t-1) e^{-n(t-1)}+A_{3}(t-1)^{2} e^{-n(t-1)}
\end{aligned} \\
& a_{0}=\alpha_{i}(1)-A_{1}, a_{1}=\left(A_{1}-A_{2}+A_{3}\right) e^{n}, a_{2}=\left(A_{2}-2 A_{3}\right) e^{n}, a_{3}=2 A_{3} e^{n}
\end{aligned}
$$

where Equation (24) is an equivalent form of Equation (23) that satisfies $\bar{\alpha}_{i}(1)=\alpha_{i}(1)$. Note that the form of Equations (23) and (24) is motivated from the analytical solution of Equation (8) with two extra polynomial terms added to account for saturation. The approximation also settles at an equilibrium value as $t \rightarrow \infty$, which matches the expected physiology. This approach follows the general philosophy presented in this paper which is to generate approximations that are valid within known physiological constraints and behaviour. Substituting three values of $t=t_{1}, t_{2}, t_{3}$ into Equation (24) yields a matrix equation:

$$
\left(\begin{array}{lll}
\beta_{11} & \beta_{12} & \beta_{13} \\
\beta_{21} & \beta_{22} & \beta_{23} \\
\beta_{31} & \beta_{32} & \beta_{33}
\end{array}\right)\left(\begin{array}{l}
A_{1} \\
A_{2} \\
A_{3}
\end{array}\right)=\left(\begin{array}{l}
b_{1} \\
b_{2} \\
b_{3}
\end{array}\right)
$$

where: 


$$
\begin{aligned}
& \beta_{j 1}=e^{-n\left(t_{j}-1\right)}-1, \beta_{j 2}=\left(t_{j}-1\right) e^{-n\left(t_{j}-1\right)}, \beta_{j 3}=\left(t_{j}-1\right)^{2} e^{-n\left(t_{j}-1\right)}, \\
& b_{j}=\alpha_{i}\left(t_{j}\right)-\alpha_{i}(1), \quad j=1, \ldots, 3
\end{aligned}
$$

Since the insulin response is evaluated over 60 minutes, $t_{3}=60$. The values of $t_{1}$ and $t_{2}$ need to be chosen carefully to take into account different insulin clearance rates $n$, and this process is explained in a later section. Equation (26) can be solved analytically for $A_{1}, A_{2}$ and $A_{3}$ in Maple which yields:

$$
\begin{gathered}
A_{3}=\frac{C_{b 1} b_{1}+C_{b 2} b_{2}+C_{b 3} b_{3}}{\operatorname{det} \beta}, A_{2}=\frac{-\beta_{21}\left(b_{1}-\beta_{3} A_{3}\right)+\beta_{11}\left(b_{2}-\beta_{23} A_{3}\right)}{C_{b 3}} \\
A_{1}=\frac{b_{1}-\beta_{12} A_{2}-\beta_{13} A_{3}}{\beta_{11}}, \operatorname{det} \beta=\operatorname{det}\left(\begin{array}{lll}
\beta_{11} & \beta_{12} & \beta_{13} \\
\beta_{21} & \beta_{22} & \beta_{23} \\
\beta_{31} & \beta_{32} & \beta_{33}
\end{array}\right) \\
C_{b 1}=\operatorname{det}\left(\begin{array}{ll}
\beta_{21} & \beta_{22} \\
\beta_{31} & \beta_{32}
\end{array}\right), C_{b 2}=-\operatorname{det}\left(\begin{array}{ll}
\beta_{11} & \beta_{12} \\
\beta_{31} & \beta_{32}
\end{array}\right), C_{b 3}=\operatorname{det}\left(\begin{array}{ll}
\beta_{11} & \beta_{12} \\
\beta_{21} & \beta_{22}
\end{array}\right)
\end{gathered}
$$

where $A_{2}$ and $A_{1}$ are defined recursively to minimise computation. Once $A_{1}, A_{2}$ and $A_{3}$ are known, $a_{0}, \ldots, a_{3}$ are determined from Equation (25). Hence the integrating factor of Equation (10) with $\alpha(t)=\alpha_{i}(t)$ in Equation (22) is approximated by:

$$
\begin{aligned}
& \mu_{i}(t)=e^{\mathrm{int} \bar{\alpha}_{i}(t)} \\
& \operatorname{int}_{\bar{\alpha}_{i}}(t)=\int_{1}^{t}\left(a_{0}+a_{1} e^{-n t}+a_{2} t e^{-n t}+a_{3} t^{2} e^{-n t}\right) d t \\
& =\operatorname{int}_{1}(t)-\operatorname{int}_{1}(1) \\
& \operatorname{int}_{1}(t)=t a_{0}+\phi_{1}(t) a_{1}+\phi_{2}(t) a_{2}+\phi_{3}(t) a_{3} \\
& \phi_{1}(t)=-\frac{1}{n} e^{-n t}, \phi_{2}(t)=-\frac{1}{n^{2}}(n t+1) e^{-n t}, \phi_{3}(t)=\frac{1}{n^{3}}\left(n^{2} t^{2}+2 n t+2\right) e^{-n t}
\end{aligned}
$$


The next step is to approximate $\bar{\mu}_{i}(t)$ in Equation (31) by:

$$
\begin{gathered}
\bar{\mu}_{i}(t)=B_{0}+B_{1} e^{D_{1} t}+B_{2} e^{D_{2} t}+B_{3} e^{D_{3} t} \\
=1+C_{1}\left(e^{D_{1}(t-1)}-1\right)+C_{2}\left(e^{D_{2}(t-1)}-1\right)+C_{3}\left(e^{D_{3}(t-1)}-1\right) \\
D_{1}=\frac{\operatorname{int}_{\bar{\alpha}_{i}}\left(t_{1}\right)}{t_{1}}, D_{2}=\frac{\operatorname{int}_{\bar{\alpha}_{i}}\left(t_{2}\right)}{t_{2}}, D_{3}=\frac{\operatorname{int}_{\bar{\alpha}_{i}}\left(t_{3}\right)}{t_{3}} \\
B_{0}=C_{0}-C_{1}-C_{2}-C_{3}, B_{1}=C_{1} e^{-D_{1}}, B_{2}=C_{2} e^{-D_{2}}, B_{3}=C_{3} e^{-D_{3}}
\end{gathered}
$$

The motivation for the form of Equation (34) comes from the fact that, when $t=t_{k}$, in Equation (34) the $k^{\text {th }}$ exponential term $e^{D_{k} t}$ becomes $e^{\text {int }_{\alpha_{i}}\left(t_{k}\right)}=\mu_{i}\left(t_{k}\right)$. Hence, each basis function $e^{D_{k} t}$ behaves like the true solution in a neighbourhood surrounding each point $t=t_{k}$, where $k=1, \ldots, 3$. the sum in Equation (34) ensures the solution is accurate between the points $t=t_{k}$. Note that a cubic could have potentially been used instead of the sum of exponentials in Equation (34) however it was found to be a very poor interpolant between the $t_{k}$ time points.

Substituting $t=t_{1}, t_{2}, t_{3}$ into Equation (35) yields a matrix equation:

$$
\left(\begin{array}{lll}
\gamma_{11} & \gamma_{12} & \gamma_{13} \\
\gamma_{21} & \gamma_{22} & \gamma_{23} \\
\gamma_{31} & \gamma_{32} & \gamma_{33}
\end{array}\right)\left(\begin{array}{l}
C_{1} \\
C_{2} \\
C_{3}
\end{array}\right)=\left(\begin{array}{l}
d_{1} \\
d_{2} \\
d_{3}
\end{array}\right)
$$

where:

$$
\begin{aligned}
& \gamma_{j_{1}}=e^{D_{1}\left(t_{j}-1\right)}-1, \gamma_{j_{2}}=e^{D_{2}\left(t_{j}-1\right)}-1, \gamma_{j_{3}}=e^{D_{3}\left(t_{j}-1\right)}-1 \\
& d_{j}=\mu_{i}\left(t_{j}\right)-1, \quad j=1, \ldots, 3
\end{aligned}
$$


Replacing $A$ with $C, \beta$ with $\gamma$ and $b$ with $d$ in Equations (28)-(30) yields the closed form solution to $C_{1}, C_{2}, C_{3}$ in Equation (38).

Given $\bar{\mu}_{i}(t)$ in Equation (34), the analytical solution to Equations (10) and (11) after 1 minute based on the integrating factor can be approximated by:

$$
\begin{aligned}
I_{i, \text { approx }}(t) & =\frac{1}{\bar{\mu}_{i}(t)}\left(I_{b, \text { approx }}(1)+u_{i} \operatorname{int}_{\bar{\mu}_{i}}(t)\right) \\
\operatorname{int}_{\bar{\mu}_{i}}(t) & =(t-1) B_{0}+\left(e^{D_{1} t}-e^{D_{1}}\right) \frac{B_{1}}{D_{1}}+\left(e^{D_{2} t}-e^{D_{2}}\right) \frac{B_{2}}{D_{2}}+\left(e^{D_{3} t}-e^{D_{3}}\right) \frac{B_{3}}{D_{3}}
\end{aligned}
$$

where Equation (41) is derived from integrating Equation (34) from $t=1$ to $t$.

\subsubsection{Iterative solution}

For a given initial insulin approximation $I_{\alpha_{I}=0}(t)$ from Equations (7)-(9), which corresponds to no saturation, Equations (20), (21) and (40) give an approximation to the solution of Equation (3) with saturation. This approximation can then be improved by replacing $I_{\text {approx }}(t)$ in Equation (11) with the piece-wise linear approximation of insulin in the first minute given by Equations (20), (21) and replacing $I_{a p p r o x}(t)$ by Equation (40) after 1 minute. Equations (20), (21) and (40) will then define a new approximation which can again be substituted into Equation (11) and so on. In practice this process could be continued until the relative percentage change is less than a tolerance. However, simulations over all physiological ranges of parameters show that only 2 iterations are necessary. Therefore the algorithm is summarised with 2 iterations as shown in Figure 1. 
Step 1: Define parameters $n, \alpha_{I}, I_{0}, u_{i}, u_{b}$. Set parameters $t_{1}, t_{2}, t_{3}$ which are used in Equations (27) and (36).

Step 2: Evaluate $I_{b}(0), I_{b}(0.5), I_{b}(1)$ from Equation (9), and $I_{\alpha_{I}=0}\left(t_{1}\right), I_{\alpha_{I}=0}\left(t_{2}\right)$, $I_{\alpha_{I}=0}\left(t_{3}\right)$ from Equations (7) and (8).

Step 3: Evaluate $\alpha_{b}(0), \alpha_{b}(0.5), \alpha_{b}(1)$ from Equation (12), and $\alpha_{i}(1), \alpha_{i}\left(t_{1}\right), \alpha_{i}\left(t_{2}\right), \alpha_{i}\left(t_{3}\right)$ from Equation (22)

Step 4: Evaluate $I_{b, a p p r o x}(0.5)$ and $I_{b, a p p r o x}(1)$ using Equations (17)-(21).

Step 5: Evaluate $\beta$ from Equation (27), $A_{1}, A_{2}, A_{3}$ from Equations (28)-(30) and thus $a_{0}, a_{1}$, $a_{2}, a_{3}$ from Equation (25)

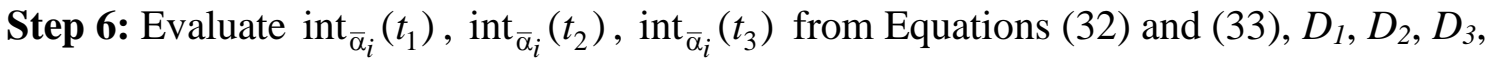
from Equation (36), $\mu_{i}\left(t_{1}\right), \mu_{i}\left(t_{2}\right), \mu_{i}\left(t_{3}\right)$ from Equation (31) and $\gamma$ from Equation (39). In Equations (28)-(30), replace $A$ with $C, \beta$ with $\gamma$ and $b$ with d to obtain $C_{1}$, $C_{2}, C_{3}$ which will solve Equation (38)

Step 7: Calculate $B_{0}, B_{1}, B_{2}, B_{3}$ from Equation (37), and thus compute $I_{i, a p p r o x}\left(t_{1}\right)$, $I_{i, \text { approx }}\left(t_{2}\right), I_{i, \text { approx }}\left(t_{3}\right)$ from Equations (40) and (41).

Step 8: Start again at Step 3 but replace $I_{b}(0.5), I_{b}(1)$ with $I_{b, a p p r o x}(0.5), I_{b, a p p r o x}(1)$ in Step 4 and replace $I_{\alpha_{I}=0}\left(t_{1}\right), I_{\alpha_{I}=0}\left(t_{2}\right), I_{\alpha_{I}=0}\left(t_{3}\right)$ with $I_{i, \text { approx }}\left(t_{1}\right), I_{i, \text { approx }}\left(t_{2}\right)$, $I_{i, \text { approx }}\left(t_{3}\right)$ in Step 7. Repeat Steps 3-7

Step 9: Output the new updates to $I_{b, \text { approx }}(0.5), I_{b, \text { approx }}(1), I_{i, \text { approx }}\left(t_{1}\right), I_{i, \text { approx }}\left(t_{2}\right), I_{i, \text { approx }}\left(t_{3}\right)$ which characterize the main features of the solution to Equation (3)

Figure 1: Algorithm for solving Equation (3) for insulin $I(t)$ 


\subsection{Analytical Solution Q(t)}

Given the discrete function evaluation, $I_{b \text {,approx }}(1), I_{i, \text { approx }}\left(t_{1}\right), I_{i, \text { approx }}\left(t_{2}\right), I_{i, \text { approx }}\left(t_{3}\right)$ from the algorithm of Figure 1, the continuous solution $I_{i, \text { approx }}(t)$ over the whole time period is first approximated by:

$$
\bar{I}_{i, \text { approx }}(t)=\bar{a}_{0}+\left(\bar{a}_{1}+\bar{a}_{2} t+\bar{a}_{3} t^{2}\right) e^{-n t}
$$

where:

$$
\begin{gathered}
\bar{a}_{0}, \bar{a}_{1}, \bar{a}_{2}, \bar{a}_{3} \equiv a_{0}, a_{1}, a_{2}, a_{3} \text { in Equation (25) with } \alpha_{i}(1) \text { replaced } I_{b, \text { approx }}(1) \\
A_{1}, A_{2}, A_{3} \equiv \text { Equations (28)-(30) with } b_{j}=I_{i, \text { approx }}\left(t_{j}\right)-I_{b, \text { approx }}(1), j=1, \ldots, 3
\end{gathered}
$$

For generality, Equation (4) is rewritten in the form:

$$
\dot{Q}=-k_{2} Q(t)+k I(t)
$$

where $k_{2}=k$ in this case.

To approximate the solution $Q(t)$ of Equation (44), $I(t)$ is defined:

$$
\begin{aligned}
I(t) & =I_{0}+\left(I_{b, \text { approx }}(1)-I_{0}\right) t, 0 \leq t \leq 1 \\
& =\bar{I}_{i, \text { approx }}(t), \quad 1 \leq t \leq 60
\end{aligned}
$$


where $I_{0}$ is the given initial condition of $I(t)$ at $t=0$. With Equation (45) and $\bar{a}_{0}, \bar{a}_{1}, \bar{a}_{2}, \bar{a}_{3}$ in Equation (43), Equation (44) can be readily solved for Q(t) in Maple which yields:

$$
\begin{gathered}
Q(t)=Q_{2}(t)+\left(Q_{1}(1)-Q_{2}(1)\right) e^{-k(t-1)}, t \geq 1 \\
Q_{2}(t)=\frac{k}{k_{2}} \bar{a}_{0}+C_{\bar{a}_{1}}(t) \bar{a}_{1}+C_{\bar{a}_{2}}(t) \bar{a}_{2}+C_{\bar{a}_{3}}(t) \bar{a}_{3} \\
Q_{1}(1)=Q_{0} e^{-k_{2}}+\frac{k}{k_{2}^{2}}\left(I_{b, \text { approx }}(1)\left(k_{2}-1\right)+I_{0}-\left(I_{0}\left(k_{2}+1\right)-I_{b, \text { approx }}(1)\right) e^{-k_{2}}\right) \\
C_{\bar{a}_{1}}=\frac{k}{k_{2}-n} e^{-n t}, C_{\bar{a}_{2}}=\frac{k\left(\left(k_{2}-n\right) t-1\right)}{\left(k_{2}-n\right)^{2}} e^{-n t}, C_{\bar{a}_{3}}=\frac{k\left(2+\left(k_{2}-n\right)^{2} t^{2}-2 t\left(k_{2}-n\right)\right)}{\left(k_{2}-n\right)^{3}} e^{-n t}
\end{gathered}
$$

Setting $k_{2}=k$ in Equations (47)-(49) gives an approximation of the solution to $Q(t)$ in Equation (2).

\subsection{Glucose solution at 60 minutes}

Equation (5) is first re-written in the form:

$$
\dot{G}_{\text {total }}+\bar{Q}(t) G_{\text {total }}=d_{0}+d_{1} e^{-k p^{t}}
$$

where:

$$
\begin{gathered}
\bar{Q}(t)=p_{G}+S_{I} \frac{Q(t)}{1+\alpha_{G} Q(t)}, d_{0}=\bar{P}_{1}, d_{1}=P(0)-\bar{P}_{1} \\
\bar{P}_{1} \equiv \text { new feed rate, } P(0) \equiv \text { old feed rate }
\end{gathered}
$$


$Q(t)$ is defined in Equations (46)-(49)

The integrating factor of Equation (49) is defined:

$$
\mu_{G}(t)=e^{\int_{0}^{t} \bar{Q}(t) d t}
$$

The interstitial compartment of Equation (2) essentially acts like a low pass filter, so that the sharp peak insulin response $I(t)$ in the blood plasma is significantly smoothed out. Thus, compared to $I(t)$ in Equation (45), a much simpler approximation of $\bar{Q}(t)$ can be defined:

$$
\bar{Q}_{\text {approx }}(t)=\bar{Q}(0)+\hat{a}_{1} t+\hat{a}_{2} t^{2}+\frac{\hat{a}_{3} t}{1+\hat{a}_{4} t}
$$

where the nonlinear term is added to approximately account for saturation. Note that a pure quartic was found to be not suitable for Equation (53) as it had significant oscillations. Higher order approximations had similar problems, and require 20+ evaluations of $\bar{Q}(t)$ and least squares to ensure a robust solution. Hence, the form of approximation in Equation (53) is important for minimizing computational cost and represents the most minimal model that gave satisfactory results.

For a given choice of $\hat{t}_{1}, \hat{t}_{2}, \hat{t}_{3}, \hat{t}_{4}$, define the values:

$$
\begin{aligned}
& \bar{Q}_{0}=\bar{Q}(0), \bar{Q}_{1}=\bar{Q}\left(\hat{t}_{1}\right), \bar{Q}_{2}=\bar{Q}\left(\hat{t}_{2}\right), \bar{Q}_{3}=\bar{Q}\left(\hat{t}_{3}\right), \bar{Q}_{4}=\bar{Q}\left(\hat{t}_{4}\right) \\
& \text { where } \bar{Q}(t) \equiv \text { Equation (51) }
\end{aligned}
$$


Setting $\bar{Q}_{\text {approx }}\left(\hat{t}_{j}\right)=\bar{Q}_{j}, j=1, \ldots, 4$ yields four equations which can be readily solved in Maple for $\hat{a}_{1}, \hat{a}_{2}, \hat{a}_{3}, \hat{a}_{4}$. The solution is:

$$
\begin{aligned}
& \hat{a}_{4}=\frac{a_{\bar{Q}_{0}} \bar{Q}_{0}+a_{\bar{Q}_{1}} \bar{Q}_{1}+a_{\bar{Q}_{2}} \bar{Q}_{2}+a_{\bar{Q}_{3}} \bar{Q}_{3}+a_{\bar{Q}_{4}} \bar{Q}_{4}}{b_{\bar{Q}_{1}} \bar{Q}_{1}+b_{\bar{Q}_{2}} \bar{Q}_{2}+b_{\bar{Q}_{3}} \bar{Q}_{3}+b_{\bar{Q}_{4}} \bar{Q}_{4}} \\
& \hat{a}_{2}=\frac{1}{\hat{a}_{4}}\left(C_{\bar{Q}_{0}} \bar{Q}_{0}+C_{\bar{Q}_{1}}\left(1+\hat{a}_{4} \hat{t}_{1}\right) \bar{Q}_{1}+C_{\bar{Q}_{2}}\left(1+\hat{a}_{4} \hat{t}_{1}\right) \bar{Q}_{2}+C_{\bar{Q}_{3}}\left(1+\hat{a}_{4} \hat{t}_{1}\right) \bar{Q}_{3}\right) \\
& \hat{a}_{3}=\frac{-1}{\hat{a}_{4}}\left(1+\hat{a}_{4} \hat{t}_{1}\right)\left(1+\hat{a}_{4} \hat{t}_{2}\right)\left(d_{\bar{Q}_{0}} \bar{Q}_{0}+d_{\bar{Q}_{1}} \bar{Q}_{1}+d_{\bar{Q}_{2}} \bar{Q}_{2}-\hat{a}_{2}\right) \\
& \hat{a}_{1}=\frac{1}{\hat{t}_{1}}\left(\bar{Q}_{1}-\bar{Q}_{0}\right)-\hat{t}_{1} \hat{a}_{2}-\frac{\hat{a}_{3}}{1+\hat{t}_{1} \hat{a}_{4}} \\
& a_{\bar{Q}_{0}}=\hat{t}_{34} \hat{t}_{14} \hat{t}_{13} \hat{t}_{24} \hat{t}_{23} \hat{t}_{21}, \quad \hat{t}_{i j}=\left(\hat{t}_{i}-\hat{t}_{j}\right),(i, j) \in\{1,2,3,4\} \\
& a_{\bar{Q}_{1}}=-\hat{t}_{2} \hat{t}_{3} \hat{t}_{4} \hat{t}_{34} \hat{t}_{24} \hat{t}_{23}, \quad a_{\bar{Q}_{2}}=\hat{t}_{1} \hat{t}_{3} \hat{t}_{4} \hat{t}_{34} \hat{t}_{14} \hat{t}_{13} \\
& a_{\bar{Q}_{3}}=\hat{t}_{1} \hat{t}_{2} \hat{t}_{4} \hat{t}_{14} \hat{t}_{24} \hat{t}_{21}, \quad a_{\bar{Q}_{4}}=-\hat{t}_{1} \hat{t}_{2} \hat{t}_{3} \hat{t}_{13} \hat{t}_{23} \hat{t}_{21} \\
& b_{\bar{Q}_{1}}=-\hat{t}_{1} a_{\bar{Q}_{1}}, \quad b_{\bar{Q}_{2}}=-\hat{t}_{2} a_{\bar{Q}_{2}}, \quad b_{\bar{Q}_{3}}=-\hat{t}_{3} a_{\bar{Q}_{3}}, \quad b_{\bar{Q}_{4}}=-\hat{t}_{4} a_{\bar{Q}_{4}} \\
& C_{\bar{Q}_{0}}=\frac{-1}{\hat{t}_{1} \hat{t}_{2} \hat{t}_{3}}, \quad C_{\bar{Q}_{1}}=\frac{-1}{\hat{t}_{1} \hat{t}_{13} \hat{t}_{21}}, \quad C_{\bar{Q}_{2}}=\frac{-1}{\hat{t}_{2} \hat{t}_{23} \hat{t}_{21}} \\
& C_{\bar{Q}_{3}}=\frac{-1}{\hat{t}_{3} \hat{t}_{13} \hat{t}_{23}}, \quad d_{\bar{Q}_{0}}=\frac{1}{\hat{t}_{1} \hat{t}_{2}}, \quad d_{\bar{Q}_{1}}=\frac{1}{\hat{t}_{1} \hat{t}_{21}}, \quad d_{\bar{Q}_{2}}=\frac{1}{\hat{t}_{2} \hat{t}_{21}}
\end{aligned}
$$

where Equations (55) are written in recursive form for computational efficiency. An approximation to Equation (52) is thus defined:

$$
\mu_{G}(t)=e^{\bar{Q}(0) t+\frac{1}{2} \hat{a}_{1} t^{2}+\frac{1}{3} \hat{a}_{2} t^{3}+\frac{\hat{a}_{3}}{\hat{a}_{4}}\left(t-\frac{1}{\hat{a}_{4}} \ln \left(1+\hat{a}_{4} t\right)\right)}
$$


A similar approach to Equations (34)-(39) could be used to create an integrable approximation to $\mu_{G}(t)$. However, the values of $S_{I}$ in Equation (51) are typically on the order of $10^{-5}$ [20], thus $\mu_{G}(t)$ increases at a significantly lower rate than $\mu_{i}(t)$ in Equation (31). Thus, it is possible to accurately represent $\mu_{G}(t)$ of Equation (58) by a low order polynomial to reduce computation. A cubic is chosen, which is defined:

$$
\begin{gathered}
\hat{\mu}_{G}(t)=\hat{b}_{0}+\hat{b}_{1} t+\hat{b}_{2} t^{2}+\hat{b}_{3} t^{3} \\
\hat{b}_{0}=1, \quad \hat{b}_{1}=\frac{a_{\mu_{1}}+a_{\mu_{2}} \mu_{2}+a_{\mu_{3}} \mu_{3}+a_{\mu_{4}} \mu_{4}}{\hat{b}_{1, \text { denom }}} \\
\hat{b}_{2}=-\left(\frac{1}{\hat{t}_{2}}+\frac{1}{\hat{t}_{3}}\right) \hat{b}_{1}+\frac{\hat{t}_{3}^{3}-\hat{t}_{2}^{2}-\hat{t}_{3}^{3} \mu_{2}+\hat{t}_{2}^{3} \mu_{3}}{\hat{b}_{2, \text { denom }}} \\
\hat{b}_{3}=\frac{-\left(1+\hat{t}_{2} \hat{b}_{1}+\hat{t}_{2}^{2} \hat{b}_{2}-\mu_{2}\right)}{\hat{t}_{2}^{3}} \\
a_{\mu_{1}}=-\hat{t}_{24} \hat{t}_{34}\left(\hat{t}_{2} \hat{t}_{3}+\hat{t}_{3} \hat{t}_{4}+\hat{t}_{2} \hat{t}_{4}\right) \hat{t}_{32}, \quad a_{\mu_{2}}=-\left(\hat{t}_{3} \hat{t}_{4}\right)^{2} \hat{t}_{34}, a_{\mu_{3}}=\left(\hat{t}_{2} \hat{t}_{4}\right)^{2} \hat{t}_{24} \\
a_{\mu_{4}}=\left(\hat{t}_{2} \hat{t}_{3}\right)^{2} \hat{t}_{32}, \hat{b}_{1, \text { denom }}=\hat{t}_{2} \hat{t}_{3} \hat{t}_{4} \hat{t}_{34} \hat{t}_{24} \hat{t}_{3}, \hat{b}_{2, \text { denom }}=\left(\hat{t}_{2} \hat{t}_{3}\right)^{2} \hat{t}_{23} \\
\mu_{2}=\mu_{G}\left(t_{2}\right), \quad \mu_{3}=\mu_{G}\left(t_{3}\right), \quad \mu_{4}=\mu_{G}\left(t_{4}\right)
\end{gathered}
$$

where $\hat{t}_{i j}$ is defined in Equation (56), $\mu_{G}(t)$ is defined in Equation (58), $\hat{b}_{1}, \hat{b}_{2}, \hat{b}_{3}$ are chosen so that $\hat{\mu}_{G}\left(\hat{t}_{j}\right)=\mu_{G}\left(\hat{t}_{j}\right), j=2, . ., 4$ and $\hat{t}_{2}, \hat{t}_{3}, \hat{t}_{4}$ are the same ponts in Equations (55)(57). Note that since $\hat{b}_{0}=1$ in Equation (59), only the three time points $\hat{t}_{2}, \hat{t}_{3}$ and $\hat{t}_{4}$ are required to uniquely determine $\hat{b}_{1}, \hat{b}_{2}, \hat{b}_{3}$. Putting $\hat{t}_{4}=60$, and solving Equation (50) by the integrating factor, gives the final solution for glucose at 60 minutes, which is defined: 


$$
\begin{gathered}
G_{\text {total }}(60)=\frac{1}{\mu_{G}(60)}\left(G_{\text {total }}(0)+\operatorname{int}_{\bar{\mu}_{G}}(60)\right) \\
\operatorname{int}_{\bar{\mu}_{G}}(60)=d_{0}\left(\hat{t}_{4}+\frac{1}{2} \hat{t}_{4}^{2} \hat{b}_{1}+\frac{1}{3} \hat{t}_{4}^{3} \hat{b}_{2}+\frac{1}{4} \hat{t}_{4}^{4} \hat{b}_{3}\right)+d_{1}\left(C_{\hat{b}_{0}}+C_{\hat{b}_{1}} \hat{b}_{1}+C_{\hat{b}_{2}} \hat{b}_{2}+C_{\hat{b}_{3}} \hat{b}_{3}\right) \\
C_{\hat{b}_{0}}=\frac{1}{k_{p}}\left(1-e^{-k p^{\hat{t}_{4}}}\right), \quad C_{\hat{b}_{1}}=\frac{1}{k_{p}^{2}}\left(1-\left(k_{p} \hat{t}_{4}+1\right) e^{-k_{p} \hat{t}_{4}}\right) \\
C_{\hat{b}_{2}}=\frac{1}{k_{p}^{3}}\left(2-\left(k_{p}^{2} \hat{t}_{4}^{2}+2 k_{p} \hat{t}_{4}+2\right) e^{-k_{p} \hat{t}_{4}}\right) \\
C_{\hat{b}_{3}}=\frac{1}{k_{p}^{4}}\left(6-\left(k_{p}^{3} \hat{t}_{4}^{3}+3 k_{p}^{2} \hat{t}_{4}^{2}+6 k_{p} \hat{t}_{4}+6\right) e^{-k_{p} \hat{t}_{4}}\right)
\end{gathered}
$$

\subsection{Adaptive choice of time points}

The time points $t_{1}, t_{2}$ in Equation (27) and (39) and $\hat{t}_{1}, \hat{t}_{2}, \hat{t}_{3}$ in Equations (55)-(61) need to be chosen carefully to maximize accuracy.

\subsubsection{Choice of $t_{1}, t_{2}$ in Equation (27) and (39)}

The time points $t_{1}, t_{2}$ in Equation (27) and (39) are associated with the decay in the insulin curve $I(t)$. The key determinant of this decay is the insulin clearance parameter $n$ in Equation (3). The insulin saturation $\alpha_{I}$, and the exogenous insulin input $u_{e x}(t)$ from Equation (6) do not significantly change the rate of decay. To enable an analytical determination of the time points $t_{1}$ and $t_{2}, \alpha_{I}$ and the parameters $u_{b}$ and $u_{i}$ in Equation (6) are defined:

$$
\alpha_{I}=0, \hat{u}_{b}=3000 \mathrm{mU} / \mathrm{min}, \hat{u}_{i}=\frac{2000}{60} \mathrm{mU} / \mathrm{min}
$$


An approximation to the insulin decay is thus defined:

$$
\hat{I}_{i}(t) \equiv \text { Equations (7)-(9), with parameters defined in Equation (65) }
$$

The steady state value of $\hat{I}_{i}(t)$ in Equation (66) is given by:

$$
\hat{I}_{i, s s}=\frac{\hat{u}_{i}}{n}
$$

The two time points $t_{1}$ and $t_{2}$ are defined:

$$
\begin{gathered}
t_{1}=1-\frac{1}{n} \ln \left(\frac{u_{i}-\hat{I}_{20 \%} n}{u_{i}-\hat{I}_{1}}\right), \quad t_{2}=1-\frac{1}{n} \ln \left(\frac{u_{i}-\hat{I}_{2 \%} n}{u_{i}-\hat{I}_{1}}\right) \\
\hat{I}_{1}=\frac{u_{b}+u_{i}}{n}-e^{-n}\left(\frac{u_{b}+u_{i}-10 n}{n}\right) \\
\hat{I}_{20 \%}=\hat{I}_{i, s s}+\frac{1}{5}\left(\hat{I}_{1}-\hat{I}_{i, s s}\right), \quad \hat{I}_{2 \%}=\hat{I}_{i, s s}+\frac{1}{50}\left(\hat{I}_{1}-\hat{I}_{i, s s}\right)
\end{gathered}
$$

where:

$$
\hat{I}\left(t_{1}\right)=\hat{I}_{20 \%}, \hat{I}\left(t_{2}\right)=\hat{I}_{2 \%}
$$

and $\hat{I}_{20 \%}\left(\right.$ or $\hat{I}_{2 \%}$ ) represent $20 \%$ (or $2 \%$ ) of the difference from the steady state value $\hat{I}_{i, s s}$ to the peak value $\hat{I}_{1}$. The value of $2 \%$ was chosen since the resulting value of $t_{2}$ corresponds quite closely to the maximum curvature in $\hat{I}(t)$, after which the curve rapidly approaches a horizontal line. The value of $20 \%$ was chosen as it corresponds to about 1.5 time constants, which occurs approximately at the point where $\hat{I}(t)$ starts 
moving away from its initial linear decay. Simulation has also confirmed that these values are optimal choices over all physiological ranges of the parameters.

The major advantage of the choice of $t_{1}$ and $t_{2}$ in Equations (68), is that they automatically account for differences in the clearance parameter $n$ which can change significantly between patients [30]. Thus, the approximation of Equation (42) is essentially independent of the clearance $n$, ensuring a robust and accurate solution, that can adapt to different patients.

\subsubsection{Choice of $\hat{t}_{1}, \hat{t}_{2}, \hat{t}_{3}$ in Equation (56)}

The time points $\hat{t}_{1}, \hat{t}_{2}, \hat{t}_{3}$ in Equation (56) are associated with the rise and decay of the interstitial insulin $Q(t)$. The key feature in $Q(t)$ which can change significantly between patients is the time of the peak. This peak time is affected by both the clearance $n$ and the effective insulin life parameter $k$ in Equations (2) and (3). Solving Equation (3) for $Q(t), 0 \leq t \leq 1$, with a linear insulin approximation $I(t)=\hat{I}_{i}(0)+\left(\hat{I}_{i}(1)-\hat{I}_{i}(0)\right) t$, and with $\hat{I}_{i}(t)$ given in Equation (66), yields an approximation to $Q(1)$ defined:

$$
\hat{Q}(1)=\frac{1}{k}\left(e^{-k}\left(\hat{I}_{i}(1)-(1+k) \hat{I}_{i}(0)+k Q_{0}\right)+\hat{I}_{i}(0)+(k-1) \hat{I}_{i}(1)\right)
$$

In Maple, solving Equations (2) and (3) for $Q(t), t \geq 1$, with the parameters in Equation (65), then solving $\dot{Q}(t)=0$ for $t$, yields an approximation to the $Q(t)$ peak time:

$$
\hat{t}_{\max }=\frac{1}{k-n} \ln \left(\frac{(k-n) Q_{1}+\hat{u}_{i}-k I_{1}}{\hat{u}_{i}-I_{1} n}\right)+1
$$

The three time points $\hat{t}_{1}, \hat{t}_{2}, \hat{t}_{3}$ in Equation (56) are equally spaced around $\hat{t}_{\max }$ which yields: 


$$
\hat{t}_{1}=\frac{\hat{t}_{\max }}{2}, \quad \hat{t}_{2}=\hat{t}_{\max }, \quad \hat{t}_{3}=\frac{\hat{t}_{\max }+60}{2}
$$

The approximation in Equation (74) accounts for significant shifts in the peak time of $Q(t)$ due to changes in $n$ and $k$. Thus, the approximation in Equation (53) automatically adapts to different parameters ensuring a robust and consistent solution to $Q(t)$.

\subsection{Summary of algorithm}

The following flow chart in Figure 2 summarises each step of the analytical algorithm which solves Equations (2)-(5) for each insulin and feed input on the hour.

\section{Input Parameters:}

$I(0), Q(0), G(0), S_{I}, P_{G}, u_{e x}(t), n, k, k_{p}, \bar{P}(0)$ and $P(0)$, where $I(0), Q(0), G(0)$ are set equal to $I(60), Q(60), G(60)$ from the prior hour.

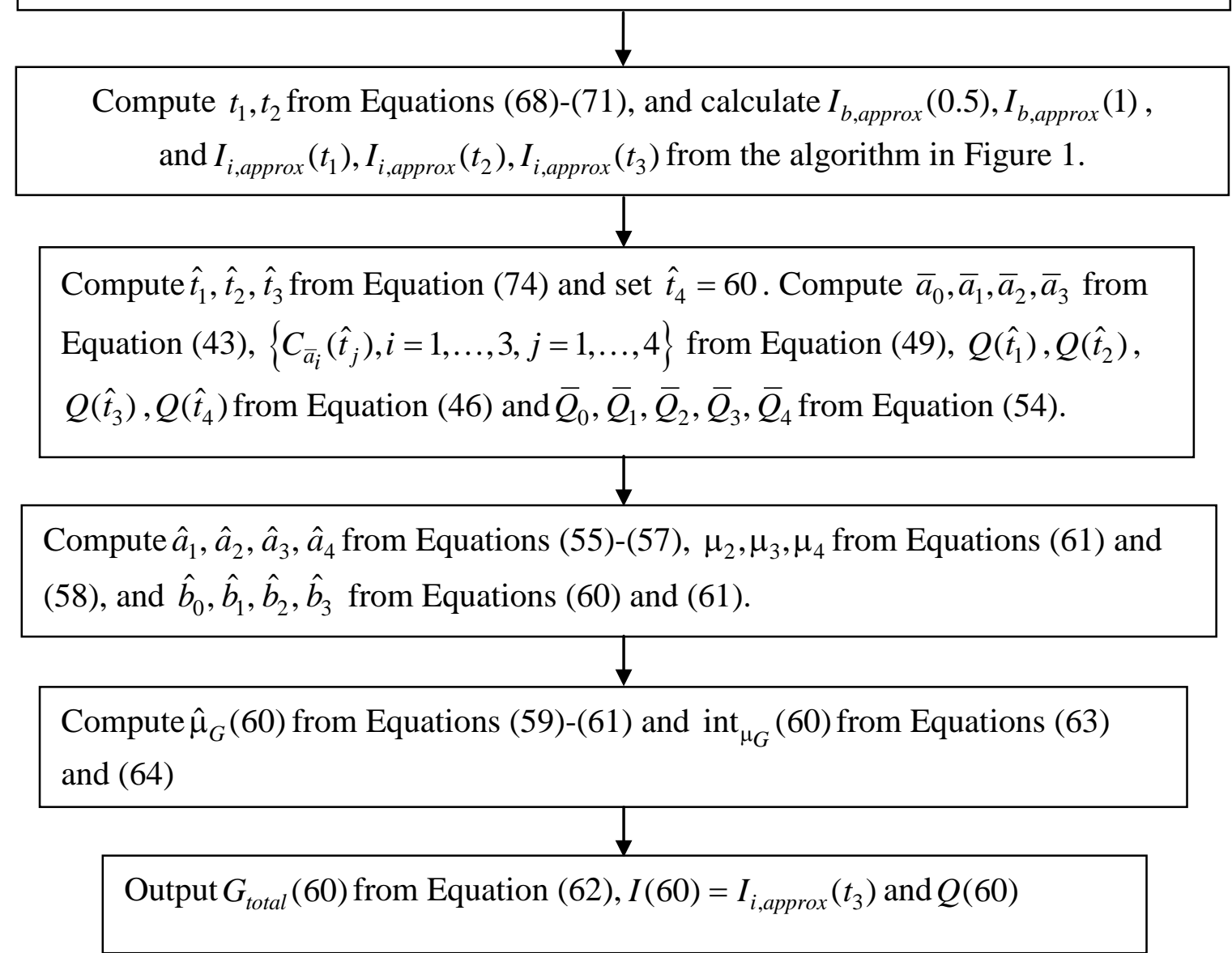

Figure 2: Algorithm for approximate analytical solution of Equations (2)-(5). 
The algorithm of Figure 2 is applied every hour in this study, to produce the glucose response from a given insulin bolus and/or infusion, nutritional infusion and identified insulin sensitivity for that hour. The parameters $n$ and $k$ are assumed constant for a given patient. Hence, a number of variables in the algorithm of Figure 2 can be pre-computed before the full simulation from hour to hour to reduce computation. These pre-computed variables are summarized:

- Pre-compute $t_{1}, t_{2}$ from Equations (68)-(71), $\beta$ from Equation (27) and $A_{1}, A_{2}, A_{3}$ from Equations (28)-(30)

- Pre-compute $\left\{\phi_{i}(1), \phi_{i}\left(t_{j}\right), i=1, \ldots, 3, j=1, \ldots, 3\right\}$ from Equation (33), to allow fast computation of $\operatorname{int}_{\bar{\alpha}_{i}}\left(t_{1}\right), \operatorname{int}_{\bar{\alpha}_{i}}\left(t_{2}\right), \operatorname{int}_{\bar{\alpha}_{i}}\left(t_{3}\right)$ from step 6 of Figure 1.

- Pre-compute $a_{\bar{Q}_{0}}, \ldots, a_{\bar{Q}_{4}}, b_{\bar{Q}_{1}}, \ldots, b_{\bar{Q}_{4}}$ from Equation (56) and $C_{\bar{Q}_{0}}, \ldots, C_{\bar{Q}_{3}}, d_{\bar{Q}_{0}}, \ldots, d_{\bar{Q}_{2}}$ from Equation (57)

- Pre-compute $a_{\mu_{1}}, \ldots, a_{\mu_{4}}, \hat{b}_{1, \text { denom }}, \hat{b}_{2, \text { denom }}$ from Equation (61) and $\left\{C_{\bar{a}_{i}}\left(\hat{t}_{j}\right), i=1, \ldots, 3, j=1, \ldots, 4\right\}$ from Equation (49)

\subsection{More general model}

The insulin Equations (2) and (3) are decoupled and can be solved independently to the glucose Equation (5). One physiological mechanism that can couple insulin to glucose is endogenous insulin production (EIP), which typically increases as blood glucose increases. In a type 2 diabetic this mechanism is still present, but is significantly less effective at controlling glucose [45]. 
The infusion of insulin is known to suppress EIP [46], and in type 1 diabetes there is no EIP. In critical care insulin is continuously infused, and thus the model of Equations (2)(5) is appropriate in these cases. However, to allow the possibility of more complex models that account for EIP-glucose coupling which could occur in non-insulin dependent diabetes, or in cases where no insulin is present, a more general model is considered. This model is also presented to demonstrate that the methods developed in this paper are generalizable. The new model is defined:

$$
\begin{aligned}
& \dot{G}=-p_{G}\left(G-G_{e}\right)-S_{I} \frac{Q(t)}{1+\alpha_{G} Q(t)} G+P(t) \\
& \dot{I}=-\frac{n I}{1+\alpha_{I} I}-n_{2}(I-Q)+\frac{u_{e x t}(t)}{V}+n_{3} G \\
& \dot{Q}=-k(Q-I)-k_{Q} Q
\end{aligned}
$$

where $P(t)$ is defined in Equation (4). The form of Equations (75)-(77) is based loosely on the physiological model of [30], but in this case EIP is assumed to be proportional to glucose to create a glucose-insulin coupling. The purpose of this model is purely to test the methods, therefore, the physiological justification and interpretation of parameters is not given here, see [30] for more details.

To allow the algorithm of Figure 2 to be used directly to solve Equations (75)-(77), the Equations of (76) and (77) are reformulated and approximated by:

$$
\begin{gathered}
\dot{I}=-\hat{\alpha}(t) I+\hat{u}_{\text {ext }}(t) \\
\dot{Q}=-k_{2} Q+k I, \quad k_{2}=k+k_{Q} \\
\hat{\alpha}(t)=\frac{n}{1+\alpha_{I} I}+n_{2}, \hat{u}_{\text {ext }}(t)=\frac{u_{\text {ext }}(t)+V\left(n_{3} G_{\text {mean }}+n_{2} Q_{\text {mean }}\right)}{V} \\
G_{\text {mean }}=\frac{1}{60} \int_{0}^{60} G(t) d t, Q_{\text {mean }}=\frac{1}{60} \int_{0}^{60} Q(t) d t
\end{gathered}
$$


where $u_{e x t}(t)$ is defined in Equation (6), and $G_{\text {mean }}$ and $Q_{\text {mean }}$ are constant approximations to $G$ and $Q$ in Equation (76). Note that, the integrals of Equation (81) are approximated by the trapezium rule and are defined:

$$
G_{\text {mean }}=\frac{G_{0}+G_{\text {total }}(60)}{2}, \quad Q_{\text {mean }}=\frac{\hat{t}_{\text {max }}\left(Q_{0}+Q\left(\hat{t}_{\text {max }}\right)\right)+\left(60-\hat{t}_{\text {max }}\right)\left(Q\left(\hat{t}_{\text {max }}\right)+Q(60)\right)}{120}
$$

where $\hat{t}_{\max }$ is defined in Equation (73). The algorithm for solving Equations (75), (78) and (79) is given in Figure 3. Note that more accurate higher order polynomial approximations to $G$ and $Q$ in Equation (76) could be readily included by re-computing Equations (15), (20), (21) and Equations (40) and (41), using $\hat{u}_{\text {ext }}(t)$ in Equation (80) rather than $u_{e x t}(t)$ in Equation (6). All other equations would remain the same. However, it has been found in simulation that constant approximations to $G_{\text {mean }}$ and $Q_{\text {mean }}$ are more than adequate for approximating the solution to Equations (75)-(77), with errors in glucose typically less than $0.4 \%$. In addition, the main purpose of this section is to show the generalizable ability of the overall approach to other glucose-insulin systems, which the example of Equations (75)-(81) achieves.

Step 1: Set $G_{\text {mean }}=G_{0}$ and $Q_{\text {mean }}=Q_{0}$ in Equation (80)

Step 2: Solve Equations (75), (78) and (79) using the algorithm of Figure 2 with $\alpha_{b}(t)$ and $\alpha_{i}(t)$ in Equations (12) and (22) replaced by $\hat{\alpha}(t)$ in Equation (80); $u_{i}$ in Equation (6) replaced by $V\left(n_{3} G_{\text {mean }}+n_{2} Q_{\text {mean }}\right)$ from Equation (80); $k_{2}$ in Equation (44) replaced by $k_{2}$ in Equation (79).

Step 3: Output $Q\left(\hat{t}_{\max }\right), Q(60)$ and $G_{\text {total }}(60)$ from Step 2

Step 4: Approximate $G_{\text {mean }}$ and $Q_{\text {mean }}$ in Equation (81) by Equation (82)

Step 5: Repeat Steps 2-4 until $G_{\text {total }}(60)$ changes by less than a given tolerance

Figure 3: Algorithm for solving (75), (78) and (79) 


\section{$3 \quad$ Results}

\subsection{Uncontrolled virtual patients}

In the retrospective trial of 17 ICU patients in [20] the patients were effectively uncontrolled due to insulin being administered "ad-hoc" by clinical staff using their own discretion and experience. The insulin sensitivity profiles obtained thus represent highly dynamic changes in patient's metabolic systems, with significantly large changes in glucose levels. These variations can be simulated by solving Equations (2)-(5), using the same insulin and nutrition inputs given by clinical staff, providing a virtual retrospective clinical trial. A virtual trial on uncontrolled patients thus provides a test of the flexibility and robustness of the analytical solution to capture highly variable physiological changes in patients, and to control them clinically.

Figure 4 shows the numerical versus analytical simulation results overlaid for Patient 87 from the ICU cohort of [20] using the recorded insulin infusion profile which contained no boluses. Also shown is the glucose measurement points with $7 \%$ error bars and the time-varying insulin sensitivity. The insulin sensitivity was found using the parameter identification method detailed in [20]. This method of identifying insulin sensitivity has been validated against the glucose clamp in clinical trials [30], hence the profile in Figure 4 is a realistic representation of the variability in the patient's physiology. For further details on the parameter identification method see [20]. The reference glucose curve in the upper panel of Figure 4 is generated by solving the full glucose model of Equations (2)-(5) for each hour with an insulin sensitivity given in the lower panel. The carbohydrate and insulin inputs were recorded by nursing staff [20] and also change every hour. Figure 4 shows that the model accurately captures the measured glucose values and the mean absolute relative percentage error between the glucose curves is $0.036 \%$ with a $90^{\text {th }}$ percentile of $0.056 \%$. These values are well within measurement errors of $7-10 \%$ and less than 100x smaller. Thus, the numerical and analytical solutions are, clinically, effectively identical. 
Note that the large oscillations in glucose are primarily caused by significant changes in patient condition which are reflected by the large changes in $S_{I}$ in the bottom panel of Figure 4. The problem is that $S_{I}$ changes before nursing staff realize so that when they react to it, $S_{I}$ can change again so they will over (or under) respond. For a full analysis of these effects and the variability that can occur in $S_{I}$, insulin dosing and carbohydrates see [44].
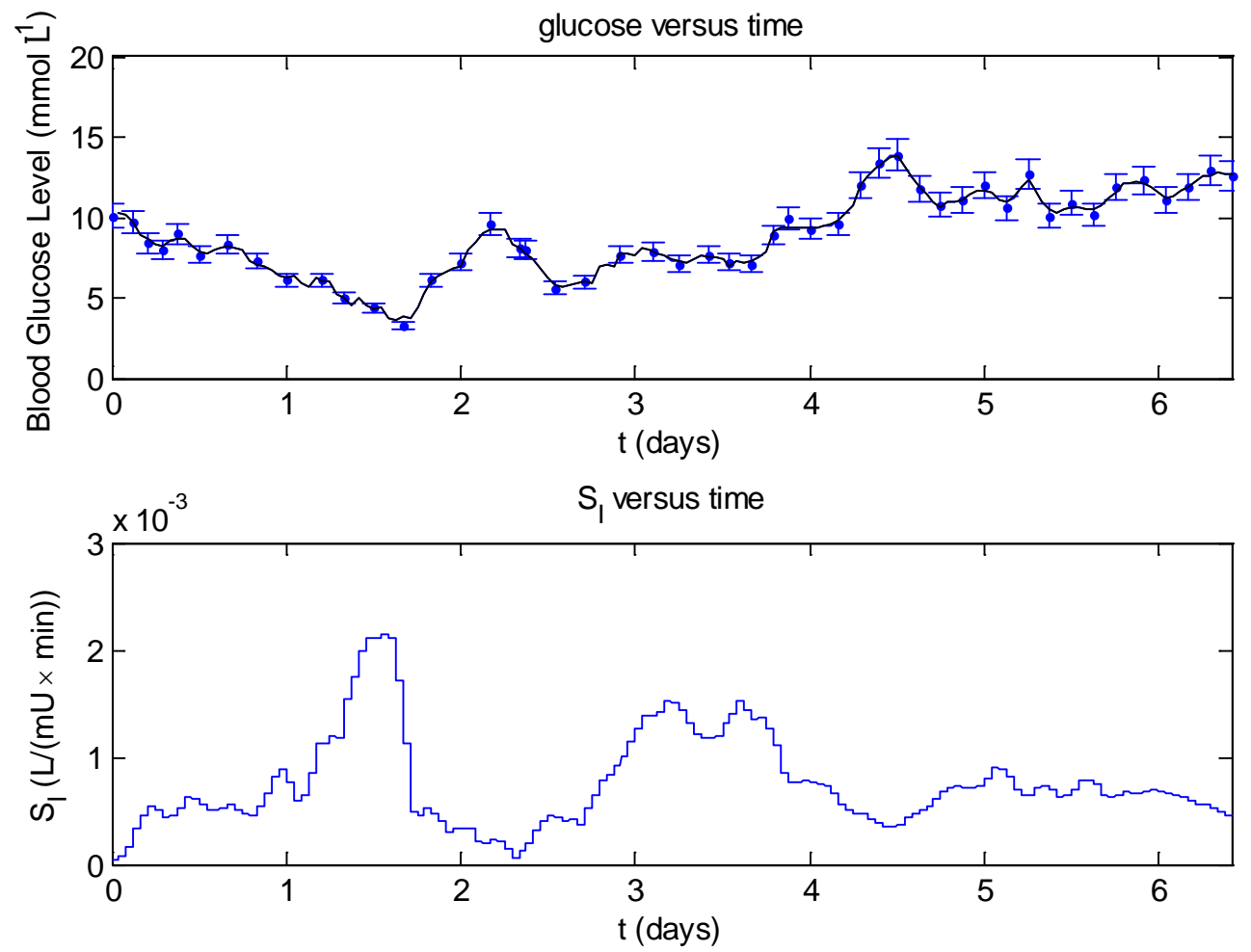

Figure 4: Numerical and analytical simulations of patient 87 [22]

Table 1 shows the statistics of the absolute relative percentage error between the numerical simulation and the analytical method described in this paper, for the entire 17 patient cohort. All patients were simulated with two different interventions. First, with only constant infusion inputs of insulin over 1 hour periods, which were the actual infusions given by the clinical staff [20]. The second intervention used a random bolus insulin input each hour of between 0 and $6 \mathrm{U}$ to further test the method. Simulations were 
compared to a standard numerical differential equation solver in Matlab using the exact same inputs. The values of $n$ and $k$ were fixed at population values throughout so that the only parameters that varied were the time varying $S_{I}(t)$ and $G_{e}(t)$, where $G_{e}(t)$ is a 12 hour moving average of the measured glucose [20]. The ranges of variability in these parameters have been validated on clinical data. Every patient has a different $S_{I}(t)$ profile, for example Figure 4 gives the $S_{I}(t)$ profile using patient 87 . For more details on how much $S_{I}$ can change for patients in an ICU, see [20].

Over the whole cohort, the mean absolute relative error was found to be very small in both cases, especially for the simulation receiving insulin through a constant infusion. The mean absolute relative error for the simulation with only bolus insulin input, was found to be slightly larger, which is due to the Michaelis Menten saturation term in Equation (3) affecting the insulin bolus input more than the constant infusion. However with a peak error of $0.09 \%$ at the $90^{\text {th }}$ percentile the error is essentially negligible and a very small fraction of the typical measurement error.

Table 1: Statistics for absolute percentage error for uncontrolled patient virtual trials

\begin{tabular}{|l|c|c|}
\hline Patient & Median (\%) & $\mathbf{9 0}^{\text {th }}$ percentile \\
\hline $\begin{array}{l}\text { Total cohort } \\
\text { Infusion only }\end{array}$ & 0.009 & 0.024 \\
\hline $\begin{array}{l}\text { Total cohort } \\
\text { bolus only }\end{array}$ & 0.035 & 0.091 \\
\hline
\end{tabular}

A random assortment of individual patient results is shown in Tables 2 and 3 for either infusion or bolus control methods. Results for these individual patients are similar to the overall results in Table 1, with the errors for the infusion based case a factor of 3-4 times smaller than that of the bolus based case. 
Table 2: Absolute \% error statistics of infusion based simulations

\begin{tabular}{|c|c|c|}
\hline Patient & Median (\%) & 90 $^{\text {th }}$ Percentile \\
\hline 2 & 0.01 & 0.016 \\
\hline 4 & 0.009 & 0.022 \\
\hline 7 & 0.009 & 0.017 \\
\hline 12 & 0.018 & 0.061 \\
\hline 15 & 0.005 & 0.011 \\
\hline
\end{tabular}

Table 3: Absolute \% error statistics of bolus based simulations

\begin{tabular}{|c|c|c|}
\hline Patient & Median (\%) & $\mathbf{9 0}^{\text {th }}$ percentile \\
\hline 2 & 0.028 & 0.073 \\
\hline 4 & 0.047 & 0.112 \\
\hline 7 & 0.027 & 0.037 \\
\hline 12 & 0.065 & 0.104 \\
\hline 15 & 0.023 & 0.082 \\
\hline
\end{tabular}

\subsection{SPRINT trial patients}

These simulations are used to show that the methods can handle the significant variations in the input of insulin and nutrition regimes that occur with SPRINT and/or in the development of new improved protocols. The first test is to compare the accuracy of the analytical method retrospectively on a 51 patient SPRINT cohort [13, 14]. First, the a priori known $S_{I}(t)$ profiles, and insulin and nutrition inputs of the 51 patients are used to forward simulate Equations (2)-(5) using both the analytical and numerical methods to assess the accuracy of this method. These values pre-define the parameters in the hourly cycle of the algorithm of Figure 2.

Table 4 shows the per patient relative absolute percentage error results between the two methods for a random selection of 5 patients. Similar results to the patients in the 17 patient ICU cohort are obtained with very minimal errors. Table 5 shows the statistics for the entire 51 SPRINT patient cohort with all errors below $0.1 \%$. 
Table 4: SPRINT patient percentage error versus numerical solution for selected patients.

\begin{tabular}{|c|c|c|}
\hline Patient & $\begin{array}{l}\text { Median } \\
(\%)\end{array}$ & $\mathbf{9 0}^{\text {th }}$ percentile \\
\hline 2 & 0.025 & 0.038 \\
\hline 20 & 0.025 & 0.047 \\
\hline 35 & 0.048 & 0.073 \\
\hline 43 & 0.022 & 0.035 \\
\hline 46 & 0.057 & 0.086 \\
\hline
\end{tabular}

Table 5: SPRINT patient percentage error versus numerical solution for the whole cohort patients.

\begin{tabular}{|c|c|c|}
\hline Patient & Median (\%) & $\mathbf{9 0}^{\text {th }}$ percentile \\
\hline $\begin{array}{c}\text { Total } \\
\text { SPRINT cohort }\end{array}$ & 0.033 & 0.064 \\
\hline
\end{tabular}

\subsection{Random changes of all parameters}

To further validate the method, all the parameters $k, n, \alpha_{G}, \alpha_{I}$ are randomly changed within the documented physiological ranges in the literature [20]:

$$
k=0.0053+0.0086 \delta, n=0.02+0.28 \delta, \alpha_{G}=0.001+0.0399 \delta, \alpha_{I}=0.0005+0.0038 \delta
$$

where $\delta$ is a uniform random variable between 0 and 1 .

The remaining parameters $G_{0}, Q_{0}, I_{0}, G_{e}, S_{I}, P_{0}, P_{1}$ are defined: 


$$
G(0)=8, Q(0)=15, I(0)=15, G_{e}=8, S_{I}=0.0005
$$

Note that, Tables 1-5 show that significant changes in the parameters of Equation (84) have little effect on the accuracy of the analytical solution method presented, so the test in this section investigates the effects of changes in the remaining parameters. 1000 monte carlo simulations were performed and the median error in the glucose value at 60 minutes was $0.046 \%$ with a $90^{\text {th }}$ percentile of $0.14 \%$. Figure 5 plots a histogram of the errors which is significantly skewed to the right, and thus shows that the method is very robust and accurate over all physiological ranges of the parameters.

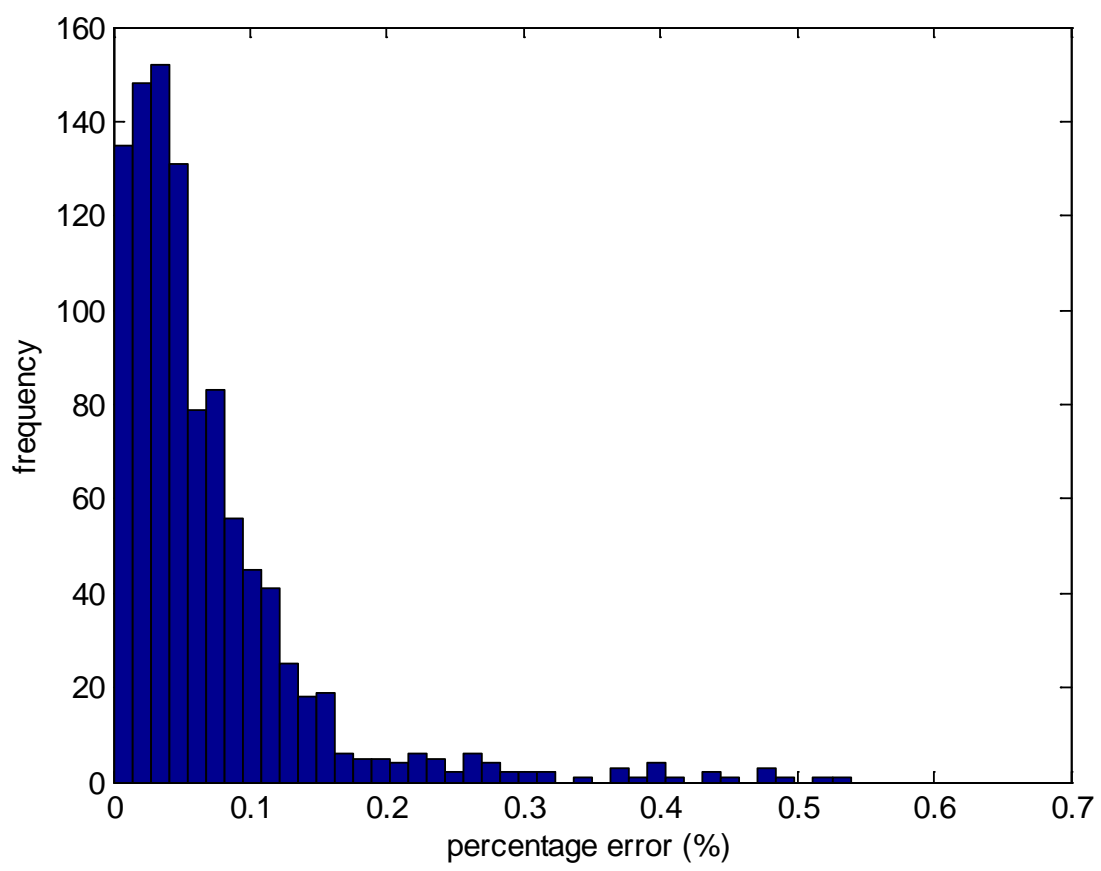

Figure 5: A histogram of all \% errors in the 1000 monte carlo simulations of the extended glucose-insulin model of Equations (75)-(77)

\subsection{Error analysis and computation speed}


The speed of the algorithm was tested on a PC with a $2.4 \mathrm{GHz}$ Pentium 4 processor and 2Gb of RAM. The original numerical simulation code used to develop the SPRINT protocol $[13,14]$ was first run using Matlab's ode45 differential equation solver. The new analytical method was then run on the same machine. Both algorithms are implemented to simulate a patient hour cycle, given predefined parameters every cycle. The overheads for inputting the parameters and storing the hourly measurement data are thus effectively identical for both simulation methods. Thus, any the differences in the processor execution time (CPU time) between methods should be independent of the input/output overheads.

The step size for Matlab's ode45 was based on an error analysis using patient 87 in Figure 4 with the same random bolus inputs used in Table 3. The "true" solution was assumed to be the numerical solution with a very small maximum relative tolerance (using odeset in Matlab) of 1e-8. A large number of tolerances were used ranging from 1e-6 to 0.001 and the $90^{\text {th }}$ percentile error over the resulting glucose response relative to the true solution is plotted in Figures 6 (a) and (b).
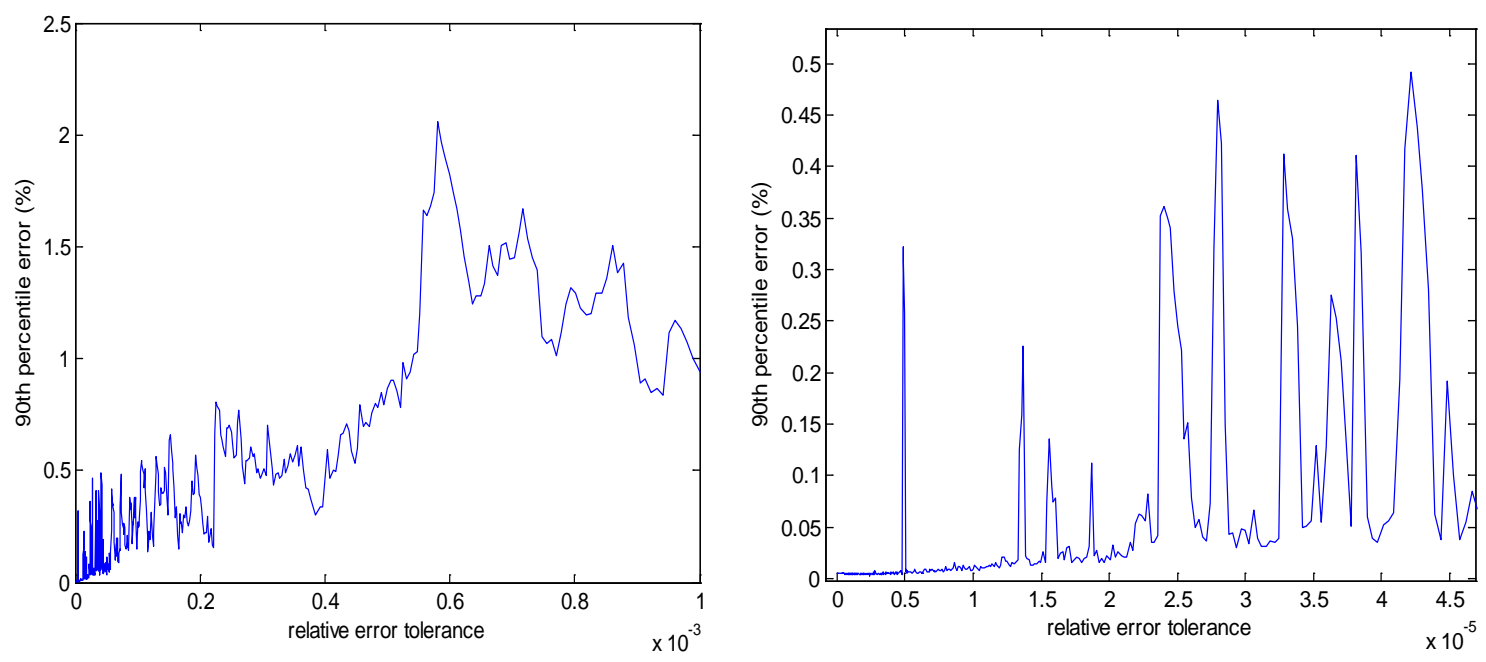

Figure 6 (a) $90^{\text {th }}$ percentile error of true versus approximated glucose for patient 87 from

Figure 4 with a range of error tolerances (b) A closer view of the error for an error tolerance less than 4.6e-5 with no clear trend in the oscillations 
As can be seen in Figure 6, the error response is very noisy and doesn't reliably settle to less than $0.1 \%$ error until the relative error tolerance is less than $0.5 \mathrm{e}-5$. Hence a value of $0.1 \mathrm{e}-5$ was chosen to ensure the $90^{\text {th }}$ percentile error is always less than $0.1 \%$. From Tables $1-5$, the analytical solution has a $90^{\text {th }}$ percentile that is typically well less than $0.1 \%$. Hence choosing the relative error tolerance of $0.1 \mathrm{e}-5$ in the numerical solver provides a fair comparison in speed between the methods. Note that the value of $1 \mathrm{e}-3$ which gives an error of $1 \%$ in Figure 6(a) is the default in Matlab and corresponds to solving the differential equation with no step-size condition specified.

As a further contrast, instead of reducing the relative error tolerance until the errors are less than $0.1 \%$, the maximum step size allowed in ode 45 is reduced. Figure 7 shows the result for maximum step sizes less than 0.2 which is the value where the $90^{\text {th }}$ percentile error is oscillating around $1 \%$. The curve is still noisy but follows a clear predictable trend of less variation as the error decreases, compared to Figure 6 which has no consistent trend and so is not as reliable. This result suggests that limiting the maximum step size is a more robust and reliable way of ensuring the error stays consistently low as compared to the faster way of reducing the relative tolerance. The dramatic differences between the responses in Figures 6 and 7 demonstrate the problem in managing error with "black box" differential equation solvers. These solvers are designed to solve every problem and hence cannot by definition, take advantage of a specific application. The approach of limiting the maximum step size was used in designing the original SPRINT system $[13,14]$, and has been found to be the most reliable way in other applications with stiff systems [36]. Hence both methods of numerical error management are considered when comparing the CPU times versus the analytical method. To keep the error below the $0.1 \%$ cut off, the maximum step size is chosen to be 0.019 . 


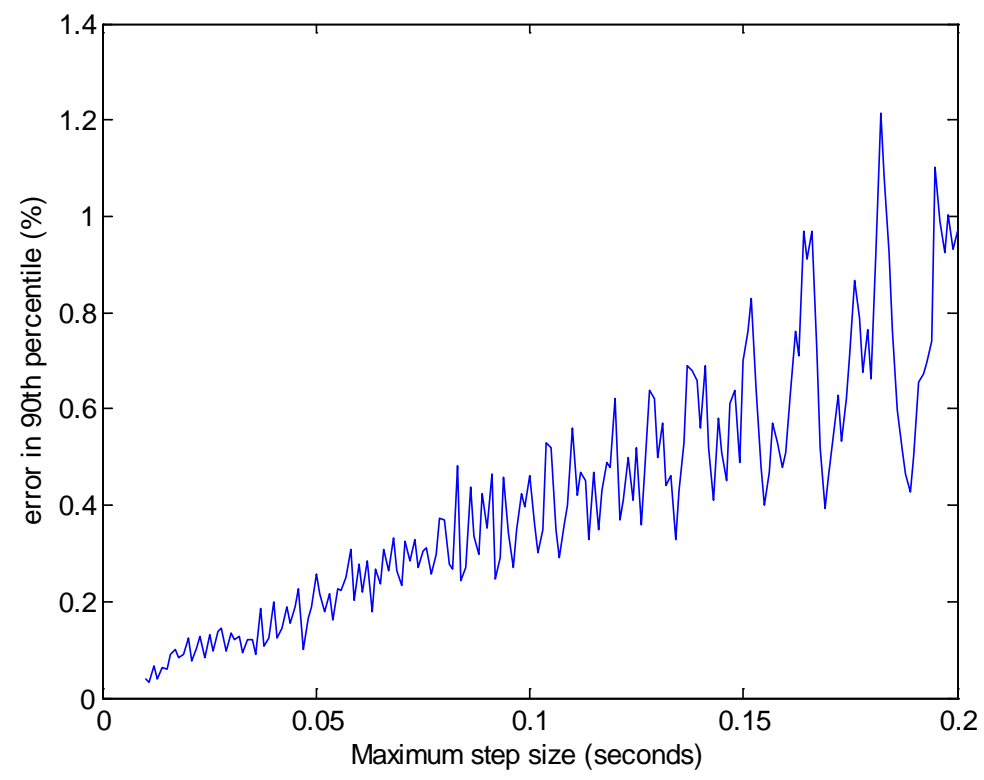

Figure 7: Plotting the maximum step size versus the $90^{\text {th }}$ percentile error in simulations of patient 87 of Figure 4. A clear trend can be seen consisting of smaller oscillations as the maximum step size decreases

Table 6 shows the CPU times for both simulation methods applied on the 51 SPRINT patient cohort. Note that the average time taken for a simulation over an hour is 6.25e-6 seconds for the analytical method, compared to 0.035 and 0.9 seconds respectively for the numerical solver. The analytical simulation is thus significantly faster, by a factor of 5600 for the relative tolerance method and a factor of 144000 for the maximum step method. Hence, for both cases, significant reductions in computational effort are made, enabling for more extensive virtual trials and Monte Carlo analyses.

Table 6: CPU times for both simulation methods on a $2.4 \mathrm{GHz}$ machine for the 51 SPRINT patient cohort

\begin{tabular}{|l|c|c|}
\hline Method & $\begin{array}{c}\text { CPU time (s) } \\
\text { [rel tol, max step] }\end{array}$ & $\begin{array}{c}\text { Factor speed increase } \\
\text { [rel tol, max step] }\end{array}$ \\
\hline Numerical simulation & {$[129,3305]$} & - \\
\hline Fast simulation & 0.023 & {$[5600,144000]$} \\
\hline
\end{tabular}




\subsection{General glucose model}

The algorithm of Figure 3 is applied to solve Equations (75)-(77). The parameters $p_{G}, n, k, \alpha_{G}, \alpha_{I}$ are taken to be the same as used for the simulations of Tables 1-5 and $G_{e}, S_{I}$ and the initial conditions are defined in Equation (84). In addition, a bolus and infusion of 1 unit are used respectively. The physiological values for the remaining parameters $n_{2}, n_{3}, k_{Q}$ are unknown, since the model of Equations (75)-(77) has not been clinically validated. Therefore, to provide a mathematical test of the concept, the values of $n_{2}$ and $k_{Q}$ are chosen randomly in the same way as $n$ and $k$ in Equation (83) and $n_{3}$ is defined as $n_{3}=0.1+1.4 \delta$, where $\delta$ is a uniform random number between 0 and 1 . Steps 2-4 in Figure 3 are performed twice in each case, as further iterations were found to not give any significant improvements.

After 1000 monte carlo simulations, the median error for this example was $0.16 \%$ with a $90^{\text {th }}$ percentile of $0.42 \%$, and no values were greater than $0.5 \%$. These errors are greater than those in Tables 1-5 and Figure 5, but this is expected since the analytical solution only solves Equations (75), (78) and (79) which is an approximation to Equations (75)(77). As stated in the formulation of this general model, more accurate approximations to $G$ and $Q$ in Equation (76) could be used if required. However, errors are still significantly less than the expected measurement error of $7 \%$, and the results demonstrate that the overall approach can be applied to more general glucose-insulin models.

\section{$4 \quad$ Discussion and Conclusions}

The concept of "Model-based Therapeutics" and the importance of fast forward simulation for enabling the rigorous testing of protocols were introduced in this paper. For a non-linear glucose-insulin model an analytical solution was created by exploiting structure and partial analytical solutions in a subset of the model, then using an iterative approach to create the final solution. Analytical and closed form solutions were always found at each stage, by writing non-integrable functions in terms of integrable function 
approximations in physiological ranges. The function approximations were made over carefully chosen time points that adapt to significant changes in the patient parameters and thus maintaining a high degree of accuracy in the results.

The key idea was to tailor the solutions to the specific application which was the rapid calculation of glucose values only on the hour, given insulin bolus, infusion and feed inputs. Current methods are numerical, which compute the glucose values at every small time step within the hour, dramatically slowing down simulation speed, and hindering the number of protocols that could be tested. The application specific approach allows parameters or parts of solutions that are unchanged for long periods, to be pre-stored further reducing computation. For example expressions involving the population insulin clearance parameters $n$ and $k$ can be separated from the analytical solution and thus calculated only once before a full monte-carlo analysis. This de-coupling of expressions from the solution cannot be done in the standard numerical approach as all parameters and values are needed at every time step.

The analytical approach gave speed increases 5600-144000 times faster than the numerical based approach depending on the error management method used in the solver. Very accurate solutions were found with $90^{\text {th }}$ percentile errors typically around $0.1-0.2 \%$ for all ranges of parameters within physiological constraints. The increased efficiency has had a significant impact on the ability to develop improved protocols, as turn around time has been massively improved and still only requires a simple programming language like Matlab. In addition, the methods developed in this paper are general and could be applied to any type of glucose-insulin model as demonstrated by the accurate results for a more complex differential equation model. This modelled coupled the glucose to insulin in the plasma and interstitial insulin compartments, and the developed algorithms were readily adapted to this situation. Future work will look at applying this concept to other areas of bio-medical engineering for example cardiac modelling [32-35] and insulin sensitivity testing [30, 39]. 


\section{$5 \quad$ References}

[1] S. Andreassen, J. J. Benn, R. Hovorka, K. G. Olesen, and E. R. Carson, "A probabilistic approach to glucose prediction and insulin dose adjustment: description of metabolic model and pilot evaluation study," Comput Methods Programs Biomed, vol. 41, pp. 153-65, Jan 1994.

[2] J. G. Chase, G. M. Shaw, X. W. Wong, T. Lotz, J. Lin, and C. E. Hann, "Modelbased Glycaemic Control in Critical Care - A review of the state of the possible," Biomedical Signal Processing and Control, vol. 1, pp. 3-21, 2006.

[3] R. Hovorka, L. J. Chassin, M. E. Wilinska, V. Canonico, J. A. Akwi, M. O. Federici, M. Massi-Benedetti, I. Hutzli, C. Zaugg, H. Kaufmann, M. Both, T. Vering, H. C. Schaller, L. Schaupp, M. Bodenlenz, and T. R. Pieber, "Closing the loop: the adicol experience," Diabetes Technol Ther, vol. 6, pp. 307-18, Jun 2004.

[4] R. Hovorka, M. E. Wilinska, and L. J. Chassin, "In Silico Simulation Environment and Glucose Control in Critically Ill Subjects: Strategic Considerations," in 3rd European Medical \& Biological Engineering Conference, Prague, Czech Republic, 2005.

[5] A. D. Morris, "Addressing dosing frequency in diabetes: A simple approach to improving adherence to therapy and clinical outcomes," Diabetes Educ, vol. 29, pp. 440-6, 448, 450-3, 2003.

[6] G. M. Steil, A. E. Panteleon, and K. Rebrin, "Closed-loop insulin delivery-the path to physiological glucose control," Adv Drug Deliv Rev, vol. 56, pp. 125-44, Feb 102004.

[7] R. S. Tudor, R. Hovorka, D. A. Cavan, D. Meeking, O. K. Hejlesen, and S. Andreassen, "DIAS-NIDDM - a model-based decision support system for insulin dose adjustment in insulin-treated subjects with NIDDM " Comput Methods Programs Biomed, vol. 56, pp. 175-192, 1998.

[8] M. Vogelzang, F. Zijlstra, and M. W. Nijsten, "Design and implementation of GRIP: a computerized glucose control system at a surgical intensive care unit," BMC Med Inform Decis Mak, vol. 5, pp. 1-10, 2005.

[9] T. S. Ahearn, R. T. Staff, T. W. Redpath, and S. I. Semple, "The use of the Levenberg-Marquardt curve-fitting algorithm in pharmacokinetic modelling of DCE-MRI data.," Phys Med Biol, vol. 50, pp. N85-92, 2005.

[10] A. Nittala, S. Ghos, D. Stefanovski, R. Bergman, and X. Wang, "Comparison of Two Compartmental Models for Describing Receptor Ligand Kinetics and Receptor Availability in Multiple Injection PET Studies," BioMedical Engineering OnLine vol. 5, pp. 44-58, 2006.

[11] G. M. Scalia, N. L. Greenberg, P. M. McCarthy, J. D. Thomas, and P. M. Vandervoort, "Noninvasive Assessment of the Ventricular Relaxation Time Constant in Humans by Doppler Echocardiography," Circulation, vol. 95, pp. 151-155, 1997.

[12] J. G. Chase, G. M. Shaw, T. Lotz, A. LeCompte, J. Wong, J. Lin, T. Lonergan, M. Willacy, and C. E. Hann, "Model-based insulin and nutrition administration for 
tight glycaemic control in critical care," Curr Drug Deliv, vol. 4, pp. 283-96, Oct 2007.

[13] T. Lonergan, A. LeCompte, M. Willacy, J. G. Chase, G. M. Shaw, X. W. Wong, T. Lotz, J. Lin, and C. E. Hann, "A Simple Insulin-Nutrition Protocol for Tight Glycemic Control in Critical Illness: Development and Protocol Comparison," Diabetes Technol Ther, vol. 8, pp. 191-206, 2006.

[14] T. Lonergan, A. L. Compte, M. Willacy, J. G. Chase, G. M. Shaw, C. E. Hann, T. Lotz, J. Lin, and X. W. Wong, "A pilot study of the SPRINT protocol for tight glycemic control in critically Ill patients," Diabetes Technol Ther, vol. 8, pp. 44962, Aug 2006.

[15] J. G. Chase, G. M. Shaw, J. Lin, C. V. Doran, C. Hann, M. B. Robertson, P. M. Browne, T. Lotz, G. C. Wake, and B. Broughton, "Adaptive bolus-based targeted glucose regulation of hyperglycaemia in critical care," Med Eng Phys, vol. 27, pp. 1-11, Jan 2005.

[16] X. W. Wong, I. Singh-Levett, L. J. Hollingsworth, G. M. Shaw, C. E. Hann, T. Lotz, J. Lin, O. S. Wong, and J. G. Chase, "A novel, model-based insulin and nutrition delivery controller for glycemic regulation in critically ill patients," Diabetes Technol Ther, vol. 8, pp. 174-90, Apr 2006.

[17] X. W. Wong, J. G. Chase, G. M. Shaw, C. E. Hann, T. Lotz, J. Lin, I. SinghLevett, L. J. Hollingsworth, O. S. Wong, and S. Andreassen, "Model predictive glycaemic regulation in critical illness using insulin and nutrition input: a pilot study," Med Eng Phys, vol. 28, pp. 665-81, Sep 2006.

[18] J. G. Chase, G. M. Shaw, A. LeCompte, T. Lonergan, M. Willacy, X. W. Wong, J. Lin, T. Lotz, D. S. Lee, and C. E. Hann, "Implementation and Evaluation of the SPRINT Protocol for Tight Glycaemic Control in Critically Ill Patients: A Clinical Practice Change," Critical Care, vol. 12, p. R49, 2008.

[19] J. G. Chase, S. Andreassen, K. Jensen, and G. M. Shaw, "The Impact of Human Factors on Clinical Protocol Performance - A proposed assessment framework and case examples," Journal of Diabetes Science and Technology (http://www.journalofdst.org), vol. 2(3), pp. 409-416, 2008.

[20] C. E. Hann, J. G. Chase, J. Lin, T. Lotz, C. V. Doran, and G. M. Shaw, "Integralbased parameter identification for long-term dynamic verification of a glucoseinsulin system model," Comput Methods Programs Biomed, vol. 77, pp. 259-270, Mar 2005.

[21] M. E. Wilinska, L. J. Chassin, and R. Hovorka, "In Silico Testing-Impact on the Progress of the Closed Loop Insulin Infusion for Critically Ill Patients Project," Journal of Diabetes Science and Technology, vol. 2, pp. 417-423, 2008.

[22] G. Van den Berghe, P. Wouters, F. Weekers, C. Verwaest, F. Bruyninckx, M. Schetz, D. Vlasselaers, P. Ferdinande, P. Lauwers, and R. Bouillon, "Intensive insulin therapy in critically ill patients," N Engl J Med, vol. 345, pp. 1359-1367, Nov 82001.

[23] J. G. Chase, C. E. Hann, G. M. Shaw, X. W. Wong, J. Lin, T. Lotz, A. J. Le Compte, and T. Lonergan, "An Overview of Glycemic Control in Critical Care Relating Performance and Clinical Results," Journal of Diabetes Science and Technology (http://www.journalofdst.org), vol. 1, pp. 82-91, 2007. 
[24] J. S. Krinsley, "Effect of an intensive glucose management protocol on the mortality of critically ill adult patients," Mayo Clin Proc, vol. 79, pp. 992-1000, Aug 2004.

[25] G. Van den Berghe, A. Wilmer, G. Hermans, W. Meersseman, P. J. Wouters, I. Milants, E. Van Wijngaerden, H. Bobbaers, and R. Bouillon, "Intensive Insulin Therapy in the Medical ICU," N Engl J Med, vol. 354, pp. 449-61, Feb. 22006.

[26] F. M. Brunkhorst, C. Engel, F. Bloos, A. Meier-Hellmann, M. Ragaller, N. Weiler, O. Moerer, M. Gruendling, M. Oppert, S. Grond, D. Olthoff, U. Jaschinski, S. John, R. Rossaint, T. Welte, M. Schaefer, P. Kern, E. Kuhnt, M. Kiehntopf, C. Hartog, C. Natanson, M. Loeffler, and K. Reinhart, "Intensive insulin therapy and pentastarch resuscitation in severe sepsis," $N$ Engl J Med, vol. 358, pp. 125-39, Jan 102008.

[27] S. Meijering, A. M. Corstjens, J. E. Tulleken, J. H. Meertens, J. G. Zijlstra, and J. J. Ligtenberg, "Towards a feasible algorithm for tight glycaemic control in critically ill patients: a systematic review of the literature," Crit Care, vol. 10, p. R19, Feb 2006.

[28] J. Plank, J. Blaha, J. Cordingley, M. E. Wilinska, L. J. Chassin, C. Morgan, S. Squire, M. Haluzik, J. Kremen, S. Svacina, W. Toller, A. Plasnik, M. Ellmerer, R. Hovorka, and T. R. Pieber, "Multicentric, randomized, controlled trial to evaluate blood glucose control by the model predictive control algorithm versus routine glucose management protocols in intensive care unit patients," Diabetes Care, vol. 29, pp. 271-6, Feb 2006.

[29] P. Devos, D. Ledoux, and J. Preiser, "Current practice of glycaemia control in european intensive care units (ICUS)," Intensive Care Med, vol. 31, p. S203, September 2005.

[30] T. Lotz, J. G. Chase, G. M. Shaw, K. A. McAuley, X. W. Wong, J. Lin, A. LeCompte, C. E. Hann, and J. I. Mann, "Monte Carlo analysis of a new modelbased method for insulin sensitivity testing," Comput Methods Programs Biomed, vol. 89, pp. 215-225, 2008.

[31] G. M. Shaw, J. G. Chase, J. Wong, J. Lin, T. Lotz, A. J. Le Compte, T. R. Lonergan, M. B. Willacy, and C. E. Hann, "Rethinking glycaemic control in critical illness - from concept to clinical practice change," Crit. Care. Resusc., vol. 8, pp. 90-9, Jun 2006.

[32] C. Hann, J. Chase, S. Andreassen, B. Smith, and G. Shaw, "Diagnosis Using a Minimal Cardiac Model Including Reflex Actions," Intensive Care Med, vol. 31, p. S18, 2005.

[33] C. E. Hann, J. G. Chase, and G. M. Shaw, "Integral-based identification of patient specific parameters for a minimal cardiac model," Comput Methods Programs Biomed, vol. 81(2), pp. 181--192, Feb 2006.

[34] G. M. Shaw, J. G. Chase, C. Starfinger, B. W. Smith, C. E. Hann, T. Desaive, and A. Ghuysen, "Modelling the cardiovascular system," Crit Care Resusc, vol. 9(3), pp. 263-269, 2007.

[35] C. Starfinger, C. E. Hann, J. G. Chase, T. Desaive, A. Ghuysen, and G. M. Shaw, "Model-based cardiac diagnosis of pulmonary embolism," Comput Methods Programs Biomed, vol. 87(1), pp. 46--60, Jul 2007. 
[36] C. E. Hann, J. G. Chase, and G. M. Shaw, "Efficient implementation of non-linear valve law and ventricular interaction dynamics in the minimal cardiac model," Comput Methods Programs Biomed, vol. 80, pp. 65-74, Oct 2005.

[37] A. D. Rudge, J. G. Chase, G. M. Shaw, D. S. Lee, and C. E. Hann, "Parameter Identification and Sedative Sensitivity Analysis of an Agitation-Sedation Model," Comput Methods Programs Biomed, vol. 83, pp. 211-221, 2006.

[38] A. D. Rudge, J. G. Chase, G. M. Shaw, and D. S. Lee, "Physiological Modelling of Agitation-Sedation Dynamics Including Endogenous Agitation Reduction," Medical Engineering \& Physics, vol. 28, pp. 629-638, 2006.

[39] T. F. Lotz, J. G. Chase, K. A. McAuley, D. S. Lee, J. Lin, C. E. Hann, and J. I. Mann, "Transient and steady-state euglycemic clamp validation of a model for glycemic control and insulin sensitivity testing," Diabetes Technol Ther, vol. 8, pp. 338-46, Jun 2006.

[40] P. Bottermann, H. Gyaram, K. Wahl, R. Ermler, and A. Lebender, "Insulin Concentrations and Time-Action Profiles of 3 Different Intermediate-Acting Insulin Preparations in Non-Diabetic Volunteers under Glucose-Controlled Glucose-Infusion Technique," Diabetes Care, vol. 5, pp. 43-52, 1982.

[41] C. Starfinger, C. E. Hann, J. G. Chase, T. Desaive, A. Ghuysen, and G. M. Shaw, "Model-based Cardiac Diagnosis of Pulmonary Embolism," Computer Methods and Programs in Biomedicine, vol. 87, pp. 46-60, 2007.

[42] R. N. Bergman, L. S. Phillips, and C. Cobelli, "Physiologic evaluation of factors controlling glucose tolerance in man: measurement of insulin sensitivity and betacell glucose sensitivity from the response to intravenous glucose," J Clin Invest, vol. 68, pp. 1456-1467, Dec 1981.

[43] R. Hovorka, F. Shojaee-Moradie, P. V. Carroll, L. J. Chassin, I. J. Gowrie, N. C. Jackson, R. S. Tudor, A. M. Umpleby, and R. H. Jones, "Partitioning glucose distribution/transport, disposal, and endogenous production during IVGTT," Am J Physiol Endocrinol Metab, vol. 282, pp. E992-1007, May 2002.

[44] C. E. Hann, J. G. Chase, M. F. Ypma, J. Elfring, N. H. MohdNor, L. P, and G. M. Shaw, "The Impact of Parameter Identification Methods on Drug Therapy Control in an Intensive Care Unit," The Open Medical Informatics Journal, vol. 2, pp. 92104, 2008.

[45] K. S. Polonsky, B. D. Given, L. J. Hirsch, H. Tillil, E. T. Shapiro, C. Beebe, B. H. Frank, J. A. Galloway, and E. Van Cauter, "Abnormal patterns of insulin secretion in non-insulin-dependent diabetes mellitus," N Engl J Med, vol. 318, pp. 1231-9, May 121988.

[46] C. H. Kim, J. Y. Park, Y. K. Shong, S. K. Hong, G. S. Kim, and K. U. Lee, "Suppression of endogenous insulin secretion by exogenous insulin in patients with insulinoma," Clinical endocrinology, vol. 52, pp. 87-92, Jan 2000. 